\title{
Learning and Information in Stochastic Networks and Queues
}

\author{
Neil Walton \\ University of Manchester \\ neil.walton@manchester.ac.uk \\ Kuang $\mathrm{Xu}$ \\ Stanford University \\ kuangxu@stanford.edu
}

October 12, 2021

\begin{abstract}
We review the role of information and learning in the stability and optimization of queueing systems. In recent years, techniques from supervised learning, bandit learning and reinforcement learning have been applied to queueing systems supported by increasing role of information in decision making. We present observations and new results that help rationalize the application of these areas to queueing systems.

We prove that the MaxWeight and BackPressure policies are an application of Blackwell's Approachability Theorem. This connects queueing theoretic results with adversarial learning. We then discuss the requirements of statistical learning for service parameter estimation. As an example, we show how queue size regret can be bounded when applying a perceptron algorithm to classify service. Next, we discuss the role of state information in improved decision making. Here we contrast the roles of epistemic information (information on uncertain parameters) and aleatoric information (information on an uncertain state). Finally we review recent advances in the theory of reinforcement learning and queueing, as well as, provide discussion on current research challenges.
\end{abstract}

\section{Introduction}

This tutorial aims to summarize the role of learning and information in queueing systems. Over the past decade, the theory of machine learning and data science has grown rapidly. As a consequence, techniques from these areas have infused themselves within a variety of operations research disciplines. The scope for applying these techniques to queueing networks is vast and important given the role of queueing networks in areas such communications, manufacturing, healthcare, supply chain and transportation. However, it is reasonable to say that the combination of learning and queueing theory is still in its infancy, particularly when compared with other areas of operations research, such as revenue management, where there are well-established connections between parameter estimation and decision making.

When we control a queueing systems, stability is usually of first order concern: can we provision service in order to cope with demand. Performance is then the next concern. It might seem that, to control a queueing system well, we need to know the arrival rate and service rate parameters, because we need to be able to solve for an optimal policy as a mapping from the state to the control action. In many practical applications, the state may not be observable, or may be costly to observe. Further errors in estimating arrival and service parameters may also incur costs. In Section 2, we look at the question of controlling a queueing system with unknown arrival rates. A classical result shows that a simple policy, called the MaxWeight policy, stabilizes the system and is agnostic to the arrival rates. We show that this policy is intimately tied to the classical result known as Blackwell Approachability, which in turn has an intimate connection with the theory of Online Convex Optimization. In Section 3, we further develop the use of online learning algorithms in queueing systems. The quality of an online 
learn algorithm is typically judged in-terms of its regret. We discuss how bounds on regret translate into bounds on the performance of a queueing model. This assesses the impact of estimating service on a queues performance. In Section 4, we assess the impact of state information on queueing network control. We discuss how, in two prior works (Spencer et al. 2014, Gamarnik et al. 2018), increased state information can dramatically improve performance.

Finally in Section 5, we review recent progress applying reinforcement learning to queueing systems. From a practical perspective, it is often possible to apply existing techniques, from reinforcement learning, to a queueing system. Indeed, as we will review, some of the earliest examples applying Q-learning with neural networks are for queueing systems. However from a theoretical perspective, the assumptions of these learning frameworks often do not naturally match with the assumptions of a queueing theoretic model. For instance, in reinforcement learning theory, it is common to assume a finite state-space and bounded rewards which are assumptions that often do not hold for a queueing systems (see Section 5.2 for further discussion). Moreover, in instances where assumptions do hold, estimation, modeling and optimization tasks of can often be treated separately. For instance, parameters of a queueing network, such as arrival rates and routing probabilities, can be estimated statistically. These can be applied to a queueing formula to estimate stationary queue lengths for a fixed capacity allocation. Given this formula service capacity can then be allocated to optimize to queue lengths. Nonetheless, there is a great deal of potential for reinforcement learning techniques to be applied to a queueing systems. Moreover, the analysis of queueing systems could greatly benefit from the more exploratory research approach taken in reinforcement learning (see Dietterich (1990)). From this there are a growing number of practical and important theoretical works emerging in this area which we highlight.

With this noted, the aim of this article is to introduce the reader to a few techniques and insights which we believe are important for understanding the role of learning and information in queueing systems, as well as potential challenges.

\subsection{Overview.}

We provide a more detailed overview of the results, discussions and approaches taken in each section of this article.

\section{Blackwell Approachability and MaxWeight.}

In Section 2, we show that a well-known stochastic network scheduling algorithms is in fact a learning algorithm. Specifically, we analyze the MaxWeight scheduling algorithm in switched queueing networks. Switched queueing networks are a discrete-time queueing network model where there are constraints on which queues can be served simultaneously (Section 2.2.1). Switched queueing networks are, for instance, used to model internet routers, wireless communication systems, data centers, and manufacturing systems. In a switch queueing network, a scheduling algorithm decides which queues are serve at each time step. In this setting, the MaxWeight algorithm is a celebrated scheduling algorithm. MaxWeight has the desirable property that it has a maximal stability region (Theorem 3). This means that, regardless of the arrival rate of jobs, if there is a way to stabilize the network then MaxWeight will stabilize the network. Importantly MaxWeight achieves this with knowledge of the underlying arrival rates.

We establish the connection between MaxWeight and Blackwell's Approachability Theorem. Blackwell's Approachability Theorem is a classical result in game theory, learning and control. Blackwell's result provides the basis for a broad class of online learning algorithms (see Abernethy et al. (2011)). Blackwell (1954) considers a game between a player and an adversary. The player receives rewards as a vector which depend on its adversaries actions. The task of the player is to make a sequence of decisions so that their average payoff converges towards a convex set, so-called Approachability. Blackwell provides a necessary and sufficient condition for approachability as well as an algorithm for the player to approach any convex set. In Proposition 1, we show that the maximal stability of MaxWeight can be seen as an instance of approachability in a game between a player who provides service and an adversary who selects arrivals. This established a direct connection between two classical results in learning and queueing network control. We note, however, that adversarial service cannot be easily be represented within this framework. This suggests that separate mechanisms are required to learn service parameters. We discuss this in Section 3. 


\section{Online Learning and Queues.}

In Section 3, we provide a simple example that assesses the impact of parameter learning on the performance a queue. A number of papers over recent years have investigated the role of learning and regret in queueing. Here we consider a model of a single server queue who must sequentially make service decisions which effect the rate of service. These service rates are unknown and so the aggregate performance of different service decisions must be predicted and optimized by an online algorithm. The performance of the online learning algorithm is typically measured in terms of its regret. The regret is the difference between the aggregate performance of the algorithm compared to aggregate performance of the best decision made in hindsight. An algorithm is deemed to have a good performance if its regret approaches zero at a fast rate. The existence regret minimizing algorithms is a consequence of Blackwell's Approachability Theorem (Theorem 2) and for this reason the theorem is considered a foundational result in online learning.

In Section 3, we provide a simple result that applies Lindley's recursion for the G/G/1 queue in order to express queueing size in-terms of the regret of the algorithm used to select service. In this way we can directly apply the regret analysis of a service rule in order to understand the impact of the algorithm on queue sizes. We apply this result for a service rule using the Perceptron Algorithm. The Perceptron Algorithm is a classical algorithm in supervised learning with allows for a particularly tractable analysis in our setting. We prove the Perceptron Mistake Bound (Theorem 4) and apply it to prove an optimality result learning a queue's service (Theorem 1).

\section{Information and Queues.}

While Section 3 demonstrates how improved parameter estimation can improve performance, Section 4 discusses how increasing state information can often be more effective. For instance, an approximate estimate of the arrival times of customers at a queue over the next 2 minutes may provide more relevant information than a highly accurate estimate of the mean arrival rate over a day. Following Lu et al. (2021), we categorize information as epistemic information and aleatoric information. Epistemic being information on underlying parameters and aleatoric information being information on the underlying system state. We review two sets of queueing theory results where the benefits of increased (aleatoric) state information can quantifiably improve performance. First we consider future information can assist in reducing delay when making admission decisions at a queue (Stidham 1985, Xu 2015). Here we see that there is a sharp cut off between an information-rich regime, which leads to marked queue size reduction, and an information-scarce regime, where performance is comparable with the best epistemic policy, i.e. the best policy that only knows parameters. Next we consider a parallel server model from Gamarnik (1998). Here we review the impacts of limited memory and limited communication. It is found that there are regimes which depend on the availability of memory and rate of messaging which, when sufficiently large, result in zero delay to arriving jobs. Again we see that ensuring sufficient state information can have a pronounced effect on performance.

There is often a natural Markovian description of a queueing system for applying performance analysis. However, these results emphasize that the state of a queueing system should not be taken as given but instead is an important consideration when designing optimization and learning techniques.

\section{Reinforcement learning and Queues.}

Finally, in Section 5, we review recent progress on the application of reinforcement learning theory to queueing systems. We note that many early examples of reinforcement learning (including early deep reinforcement learning) have been applied to scheduling problems in queueing systems. However, while it is possible to apply reinforcement learning algorithms to queueing systems in a relatively straightforward manner, we note that many of the theoretical assumptions that guarantee convergence and correctness in reinforcement learning tend not to hold in the case of a queueing system. We itemize and discuss these issues. We identify key areas where more theoretical development is required. We then provide a review of recent literature that strives to address this challenge. This includes a number of recent work at the Reinforcement Learning in Networks and Queues workshop at the 2021 ACM Sigmetrics conference.

While applications of reinforcement learning to queueing systems are still very nascent, our aim is that this tutorial provides an introduction that is both instructive but also acts as a guide on recent literature and potential themes of future research. 


\subsection{Organization.}

The remainder of the paper is organized as follows. In Section 2 we prove the relationship between the MaxWeight policy and Blackwell's Approachability Theorem. In Section 3, we analyse the regret of queueing systems when service rates are unknown. In Section 4, we discuss the use of detailed state information and its impact on performance. In Section 5, we review the recent progress applying reinforcement learning theory to queueing systems.

\section{Blackwell Approachability and MaxWeight.}

In this section, we relate classical approaches to learning, control and queueing. Specifically, we discuss connections between Blackwell's Approachability Theorem and the MaxWeight policy.

We start by presenting Blackwell's Approachability Theorem (Theorem 5). As we will discuss in more detail shortly. Blackwell's Approachability Theorem gives conditions for the time average value of a controlled random walk to approach a convex set in the setting where the random walk may be perturbed adversarially (see Section 2.1.2). The result is considered to be foundational in online convex optimization, as a number of algorithms and approaches can viewed as consequence of Blackwell's result. Specifically Blackwell (1956), proves a result on the regret of online algorithms called the Hannan-Gaddum Theorem is a corollary of his theory (Theorem 2). The regret of an online algorithm is difference between the reward of the algorithm and the reward best fixed parameter choice in hindsight (cf. (7)).

We then introduce the MaxWeight policy and prove its stability for subcritical queueing networks. Finally, we prove that the stability of MaxWeight can be viewed as a consequence of Blackwell's Approachability Theorem.

\subsection{Blackwell Approachability}

Blackwell's Approachability Theorem is a foundational result in game theory, learning and control. The result of Blackwell (1956) gives a necessary and sufficient condition for the mean of a vector payoff to approach a convex set under adversarial perturbations. The result is a generalization of the Minimax Theorem for two-person zero-sum games. In a subsequent note, Blackwell (1954) observed that this approachability property can be used to prove sub-linear regret of sequential decision policies. Below we present the model setting and then state Blackwell's Approachability Theorem; a proof of this result is provided in the appendix.

\subsubsection{The Minimax Theorem.}

Blackwell's Approachability Theorem is an extension of the Minimax Theorem, see Morgenstern and Von Neumann (1953). So we start by briefly discussing the Minimax Theorem. The Minimax Theorem applies to twoperson zero-sum games, here you make a decision $d \in \mathcal{D}$ and an adversary makes a decision $a \in \mathcal{A}$. (Here $\mathcal{D}$ and $\mathcal{A}$ are closed, bounded, convex subsets of $\mathbb{R}^{p}$.) For decision $d$ and adversary decision $a$, you receive a reward $d^{\top} R a$ and the adversary receives a cost of $d^{\top} R a$, where $R$ is a $p \times p$ real-valued matrix. Given that you want to maximize reward whiles the adversary wishes to minimize costs, the Minimax Theorem states that

$$
\min _{a \in \mathcal{A}} \max _{d \in \mathcal{D}} d^{\top} R a=\max _{d \in \mathcal{D}} \min _{a \in \mathcal{A}} d^{\top} R a
$$

The value, $v$, of the expression above is called the value of the game. Notice the inequality $\min _{a \in \mathcal{A}} \max _{d \in \mathcal{D}} d^{\top} R a \geq$ $v$ states that if we know $a$ then we can make a decision that gets at least value $v$. While $\max _{d \in \mathcal{D}} \min _{a \in \mathcal{A}} d^{\top} R a \geq$ $v$ gives the seemly stronger statement that there exists a decision the regardless of $a$ we can get at least value $v$. In Blackwell's terminology this means that the decision maker can approach the set $[v, \infty)$. Blackwell's Approachability Theorem generalizes this notion to a game with a vector of rewards, and the notion of approachability to convex sets.

\subsubsection{A vector valued game.}

At time $t \in \mathbb{N}$, a player makes a decision $d(t) \in \mathcal{D}$ and its adversary makes a decision $a(t) \in \mathcal{A}$. (Here $\mathcal{D}$ is a closed, bounded, convex subset of $\mathbb{R}^{p}$ and $\mathcal{A}$ is closed, bounded, convex subset of $\mathbb{R}^{p}$.) From this an 
$n$-dimensional vector of payoff is made

$$
R(d(t), a(t))=\sum_{i=1}^{p} \sum_{j=1}^{q} d_{i}(t) R_{i j} a_{j}(t)
$$

where, for each $i$ and $j, R_{i j} \in \mathbb{R}^{n}$. We let $R_{\max } \geq \max _{i j}\left\|R_{i j}\right\|_{2}$. Decisions from the player and adversary at each time may be a function of previous decisions and payoffs. We let $\mathcal{F}_{t}$ denote the decisions and payoffs up until time $t$. We allow $d(t)$ and $a(t)$ to be random but must be conditionally independent given the past payoffs and decisions, $\mathcal{F}_{t-1}$. We let $\bar{a}(t)=\mathbb{E}\left[a(t) \mid \mathcal{F}_{t-1}\right]$ and $\bar{d}(t)=\mathbb{E}\left[d(t) \mid \mathcal{F}_{t-1}\right]$. Starting from some initial position $Q(0) \in \mathbb{R}^{q}$, the average payoff vector is

$$
\bar{Q}(t)=\frac{1}{t}\left[Q(0)+\sum_{s=1}^{t} R(d(s), a(s))\right] .
$$

Without loss of generality, we assume that $Q(0)$ is chosen so that $\|Q(0)\|_{2} \leq R_{\max }$.

The task of the player is to make a sequence of decisions $\{d(t)\}_{t=1}^{\infty}$ such that the mean payoff $\bar{Q}(t)$ converges towards a closed, convex set $\mathcal{Z} \subset \mathbb{R}^{n}$, regardless of decisions of the adversary $\{a(t)\}_{t=1}^{\infty}$. We say that the set $\mathcal{Z}$ is approachable if this convergence is possible regardless of the sequence of decisions made by the adversary. Specifically, for all sequences $\{a(t)\}_{t=1}^{\infty}$ there exists a sequence $\{d(t)\}_{t=0}^{\infty}$ such that

$$
D(\bar{Q}(t), \mathcal{Z}) \underset{t \rightarrow \infty}{\longrightarrow} 0
$$

where $D(\bar{Q}, \mathcal{Z})$ gives the $L^{2}$-norm of the distance between $\bar{Q}$ and $\mathcal{Z}$ :

$$
D(\bar{Q}, \mathcal{Z}):=\sqrt{\mathbb{E}\left[\min _{z \in \mathcal{Z}}\|\bar{Q}-z\|^{2}\right]}
$$

\subsubsection{Blackwell's Approachability Theorem.}

Blackwell's Approachability Theorem provides necessary and sufficient conditions for approachability to hold.

Theorem 1 (Blackwell's Approachability Theorem). The following are equivalent

1. $\mathcal{Z}$ is approachable.

2. For every $q$ there exists $p$ such that $R(p, q) \in \mathcal{Z}$.

3. Every half-space containing $\mathcal{Z}$ is approachable.

We focus on the equivalence between Parts 1 and 3, above, and, for reasons of brevity, we assume that the equivalence between Parts 1 and 2 has already been shown. We note that approachability of half-spaces can be shown to be equivalent to the classical Minimax Theorem. Further we note that Blackwell's proof that Part 3 implies Part 1 is constructive, and the algorithm applied is as follows.

We define $P(t)$ to be the projection with respect to the Euclidean norm of $\bar{Q}(t)$ onto $\mathcal{Z}$. We let

$$
\begin{aligned}
\hat{n}(t) & :=\bar{Q}(t)-P(t) \\
v(t) & :=P(t) \cdot(\bar{Q}(t)-P(t)) .
\end{aligned}
$$

This defines a hyperplane

$$
\mathcal{H}(t):=\left\{r \in \mathbb{R}^{n}: \hat{n}(t) \cdot r \leq v(t)\right\} .
$$

Note that $\mathcal{Z}$ is contained in $\mathcal{H}$. Blackwell proposes to choose a random variable $d(t)$

$$
\mathbb{E}[R(d(t), a)] \in \mathcal{H}(t), \quad \forall a \in \mathcal{A} .
$$

The existence of this choice $d(t)$ is not obvious at this point, but this will follow as a consequence of the Minimax theorem. 


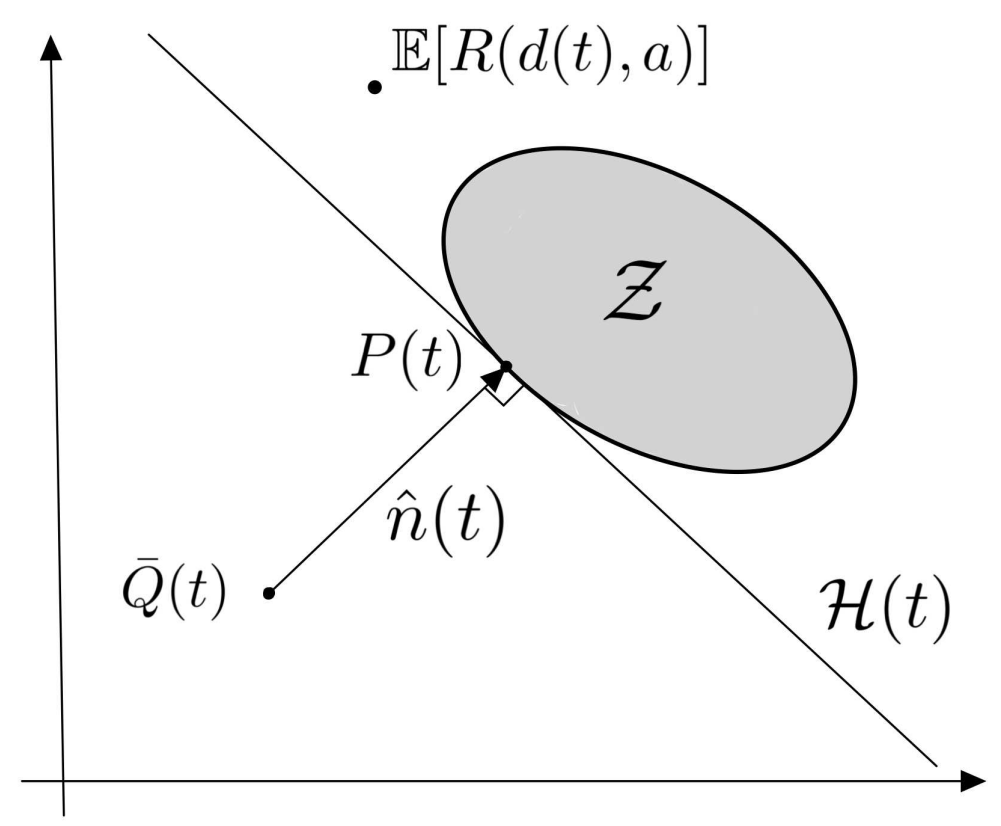

Figure 1: Blackwell's Approachability Theorem: for $\mathcal{Z}$ to be approachable, we require $\mathbb{E}[R(d(t), a)]$ to belong to the hyperplane $\mathcal{H}(t)$ for each $a \in \mathcal{A}$. Here $\mathcal{H}(t)$ is the hyperplane constructed from projecting $\bar{Q}(t)$ onto $\mathcal{Z}$.

\subsubsection{Sublinear Regret as an application of Blackwell Approachability.}

The regret minimization framework is by now standard for the analysis of online learning algorithms, online convex optimization and sequential decision making. Again here there are decisions that can be made over time and that receive rewards (or losses). The regret, which we define below, is measure the performance of a sequence of decisions against the best fixed decision. Literally, how much we regret not making the best decision in hindsight. A "good" algorithm should have regret that grows sub-linearly with time. This essentially implies that the algorithm is able asymptotically learn the best decision. The existence of sub-linear regret algorithms is a consequence of approachability, see Blackwell (1954). This connects Blackwell's Approachability Theorem with a rich stream of literature that encompasses bandit algorithms (see Lattimore and Szepesvári (2020)) and modern neural network optimizers like AdaGrad (see Hazan (2019)).

To review Blackwell's result here, we consider the following setting: again, a player makes a decision $d(t) \in \mathcal{D}$ and its adversary makes a decision $a(t) \in \mathcal{A}$. The decision set $\mathcal{D}$ is assumed to be the set of probability distributions on actions indexed by $i=1, \ldots, p$. The player receives a reward $r(i, a(t))$ for choosing index $i=1, \ldots, p$. Consequently, the reward for distribution $d(t)$ is

$$
r(d(t), a(t)):=\sum_{i=1}^{p} d_{i}(t) r(i, a(t)) .
$$

The regret of the sequence $\{d(t)\}_{t=1}^{T}$ is

$$
\mathcal{R} g(T):=\max _{i=1, \ldots, p} \sum_{t=1}^{T} r(i, a(t))-\sum_{t=1}^{T} r(d(t), a(t)) .
$$

A good algorithm should have low regret regardless of the sequence $\{a(t)\}_{t=1}^{T}$. Notice that if $\mathcal{R g}(T) \leq 0$ holds, then the performance of the algorithm is at least as good as the best fixed choice in $\mathcal{D}$. Further if $\lim \sup _{T \rightarrow \infty} \mathcal{R} g(T) / T \leq 0$ then we can see that the algorithm is in effect learning the best action. Such algorithms are referred to as being Hannan consistent, see Cesa-Bianchi and Lugosi (2006).

One interesting consequence of Blackwell's Approachability Theorem is that it can be used to construct an algorithm that is Hannan consistent. 


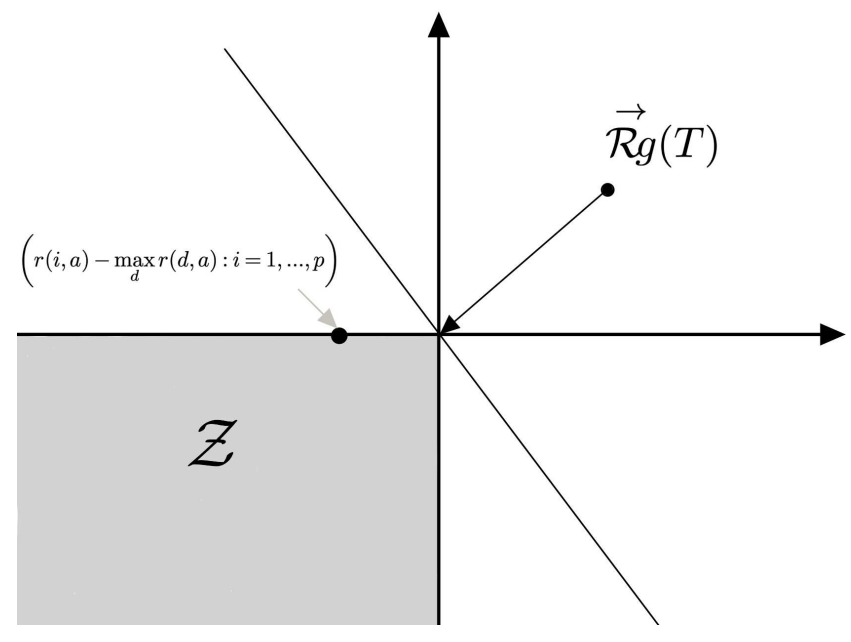

(a) Approachability for Regret

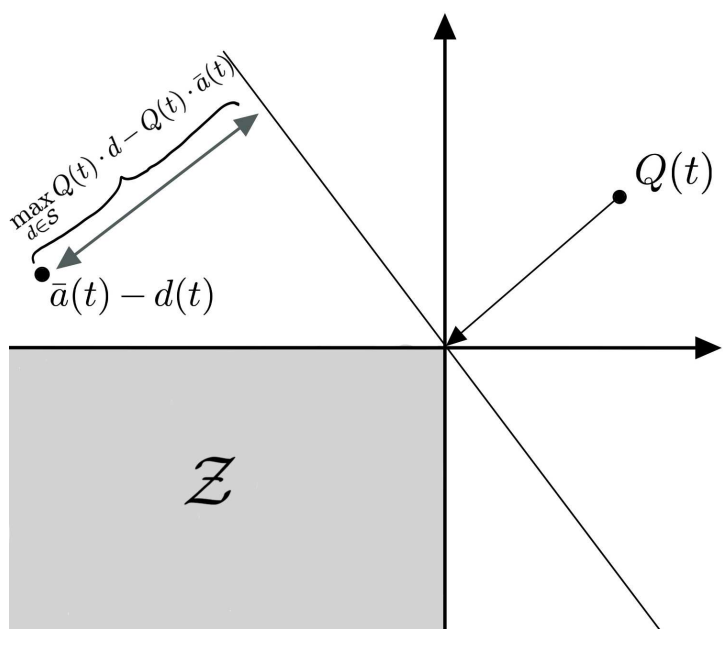

(b) Approachability for Queues

Figure 2: (a) Approachability for Regret : Here $\mathcal{Z}$ is the negative orthant. Since for each $a$ there is a decision $d$ (specifically, the maximizer of $r(d, a)$ ) such that reward difference belongs to $\mathcal{Z}$. Thus, the set $\mathcal{Z}$ is approachable. (b) Approachability for Queues: Since for each $a$ there is a decision $d$ (specifically, the maximizer of $Q(t) \cdot d)$ such that reward difference belongs is to the right of the hyperplane with normal $Q(t)$.

Theorem 2 (Hannan-Gaddum Theorem). There exists a playing strategy $\left\{d_{t}\right\}_{t=1}^{\infty}$ such that for any $\left\{a_{t}\right\}_{t=1}^{\infty}$

$$
\limsup _{T \rightarrow \infty} \mathbb{E}\left[\frac{\mathcal{R} g(T)}{T}\right] \leq 0 .
$$

In other words, our performance in the game is asymptotically as good as the best fixed action.

The result above shows that regardless of the choices of the adversary, there are always ways to learn the best fixed decision. A formal proof of this result is given in the appendix. However, the result holds essentially as follows. The regret will go to zero if each component of the following regret vector approaches zero. Specifically, if we define

$$
\overrightarrow{\mathcal{R} g}(T)=\left(\sum_{t=1}^{T} r(i, a(t))-\sum_{t=1}^{T} r(d(t), a(t)): i=1, \ldots, p\right)
$$

and $\mathcal{Z}$ is the negative orthant. I.e. $D(\overrightarrow{\mathcal{R} g}(T), \mathcal{Z}) \rightarrow 0$ as $T \rightarrow \infty$. For this problem, $R(d, a)=(r(i, a)-$ $r(d, a): i=1, \ldots, p)$. Notice that for any decision by the adversary $a$ we can chose a decision $d$ so that $R(d, a) \in \mathcal{Z}$. This verifies condition (2) in Blackwell's Approachability Theorem and so the result holds. See Figure 3 for an illustration of this proof.

\subsection{Stability and Approachability in MaxWeight}

A classical approach to scheduling in queueing networks is the MaxWeight algorithm, which was first introduced in the seminal work of Tassiulas and Ephremides (1990). In that paper, it is proved that the MaxWeight scheduling algorithm has the property of stabilizing a queueing network whenever it is possible to construct a stabilizing policy - a statement that is somewhat similar to the statement of Blackwell's result. Moreover, Neely et al. (2008) extends the framework to the optimization of different utility function objectives and Neely (2010b) extends the framework to adversarial arrivals. Below we discuss how MaxWeight can be interpreted as special case of Blackwell's policy (6). Thus the desirable stability properties of MaxWeight can be seen as a consequence of approachability.

Below, we present the queueing network setting and the MaxWeight policy. Afterwards we present the main stability result for MaxWeight; a proof of the result is provided in the appendix. Following this, we make the connection with Blackwell's Approachability Theorem.

\subsubsection{Switched Queueing Network and MaxWeight.}

We consider a discrete time queueing network, called a switched queueing network. In this model, there are constraints on which queues can be served simultaneously. We let $j=1, \ldots, q$ index the set of queues. 


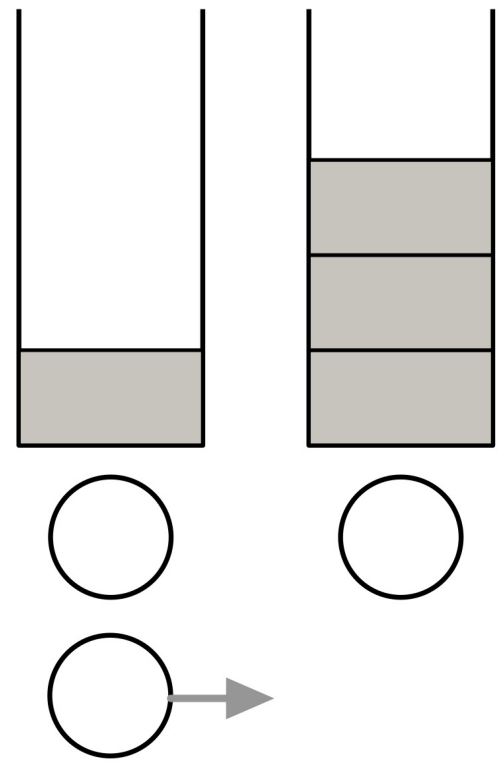

(a) Two queues with an additional flexible server

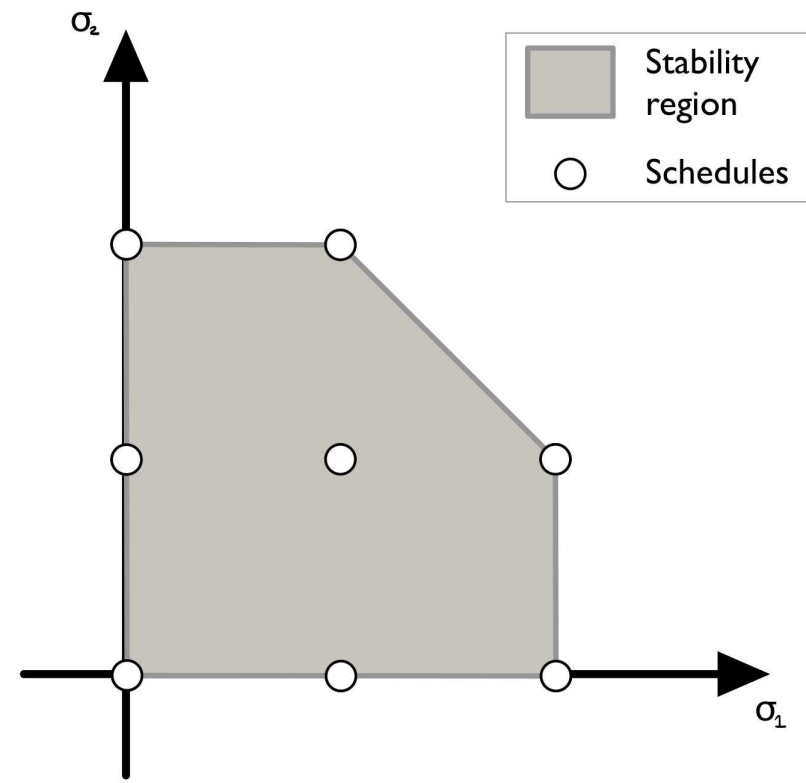

(b) Scheduling set and stability region

Figure 3: (a) Switched queueing network example: each queue has one devoted server and there is an additional server that can provide one additional unit of service to one queue only. (b) Stability Region example: the schedules for the switch network in (a) are circled; the stability region, which is the convex combination of schedules, is shaded.

At time $t \in \mathbb{Z}_{+}$, the number of arrivals to each queue is given by the vector $a(t)=\left(a_{j}(t): j=1, \ldots, q\right) \in$ $\mathbb{Z}_{+}^{q}$. For simplicity, we suppose that the components above are bounded by some maximum value $a_{\max }$. The vector $a(t)$ may be random, in which case, we let $\bar{a}(t)=\left(\bar{a}_{j}(t): j=1, \ldots, q\right)$ be the expectation of $a(t)$. We let $d(t)=\left(d_{j}(t): j=1, \ldots, q\right)$ be the number of departures for each queue at time $t$. We suppose that $d(t)$ belongs to some set of schedules $\mathcal{S} \subset\{0,1\}^{q} .^{1}$ Again we suppose that the elements of $d(t)$ are bounded by some maximum value $d_{\max }$ and that each queue can be served by some element $d$ in $\mathcal{S}$. Further, we suppose that the set of schedules $\mathcal{S}$ is monotone, meaning that for any $\sigma \in \mathcal{S}$ and $\sigma^{\prime} \in \mathbb{Z}_{+}^{q}$ if $\sigma^{\prime} \leq \sigma$ component-wise then $\sigma^{\prime} \in \mathcal{S}$. We let $\langle\mathcal{S}\rangle$ be the convex closure of $\mathcal{S}$ and we let $\langle\mathcal{S}\rangle^{\circ}$ be the interior of $\langle\mathcal{S}\rangle$.

From some initial queue size vector $Q(0)=\left(Q_{j}(0): j=1, . ., q\right)$, we can define the queueing process

$$
Q_{j}(t+1)=Q_{j}(t)+a_{j}(t)-d_{j}(t), \quad j=1, \ldots, q,
$$

for $t \in \mathbb{Z}_{+}$. (Note by our monotonicity property on $\mathcal{S}$, we may assume that $d_{j}(t)=0$ whenever $Q_{j}(t)=0$ and more generally that $d_{j}(t) \leq Q_{j}(t)$ so that queues remain positive.)

The MaxWeight policy is a well-known scheduling rule for choosing the departure vector $d(t)$ among the set of schedules $\mathcal{S}$. Specifically, at time $t$, MaxWeight chooses

$$
d(t) \in \underset{\sigma \in \mathcal{S}}{\operatorname{argmax}} \sum_{j=1}^{q} Q_{j}(t) \sigma_{j} .
$$

We assume that if a queue is empty, i.e. $Q_{j}(t)=0$, then $d(t)$ is chosen above so that $d_{j}(t)=0$. Any other ties may be broken arbitrarily. Note that queues always remain non-negative under MaxWeight. Since the scheduling decision is a function of the current queues sizes, the resulting queue size process under the recursion (9) is a Markov chain. Thus we can discuss the positive recurrence and transience of this Markov chain. When the queueing network is positive recurrent then we often refer to the network being stable, whereas a transient network is referred to as unstable.

\subsubsection{Maximal Stability of MaxWeight.}

A well-known result due to Tassiulas and Ephremedes is the following

\footnotetext{
${ }^{1}$ Many of the arguments discussed below likely extend to the case $\mathcal{S} \subset Z_{+}^{q}$. However, the assumption $\mathcal{S} \subset\{0,1\}^{q}$ simplifies our arguments when a scheduling decision empties a queue.
} 
Theorem 3. Given $a(t), t \in \mathbb{Z}_{+}$are independent identically distributed with mean $\bar{a}$ then

i) If $\bar{a} \notin<\mathcal{S}>$ then, regardless of the policy used, $\left(Q_{j}(t): j=1, \ldots, q\right)$ is transient.

ii) If $\bar{a} \in<\mathcal{S}>^{\circ}$ then, under the MaxWeight policy, $\left(Q_{j}(t): j=1, \ldots, q\right)$ is positive recurrent.

The first part of the theorem, above, establishes that $\langle\mathcal{S}\rangle$ is the maximal stability region for the switched queueing network. That is the set $\langle\mathcal{S}\rangle$ is the largest set of arrival rates for which we can hope to find a policy to stabilize the queueing network. (Note that, in principle, this policy may need to know the arrival rates to stabilize the network.) What the second part shows is that there is a single policy, specifically MaxWeight, that achieves the maximal stability region, regardless of the which arrival rate it is inside the stability region. ${ }^{2}$ We provide a sketch of part ii) below:

Proof. (Sketch Proof of Theorem 3ii).) The change in service queue size is given by the number of arrivals minus departures. We can thus approximate this with the following ordinary differential equation:

$$
\frac{d Q_{j}}{d t}=\bar{a}_{j}-\sigma_{j}(t), \quad j=1, \ldots, q, \quad t \in \mathbb{R} .
$$

Before analyzing this o.d.e., let's briefly introduce some notation. We let $Q^{\Sigma}$ be the sum of all queue sizes and we let $P_{j}$ be the proportion of jobs in queue $j$, so $Q_{j}=Q^{\Sigma} P_{j}$. Also since $\bar{a}$ belongs to the interior of $\langle\mathcal{S}\rangle$ there exists some $\epsilon>0$ such that

$$
\bar{a}+\epsilon \mathbf{1} \in\langle\mathcal{S}>\text {. }
$$

Now, for the above o.d.e. model, we can assume that the system is stable if the (euclidean) distance of the queue sizes goes to zero. We can analyze the change in this distance as follows

$$
\begin{aligned}
\frac{d\|Q\|^{2}}{d t} & =2 \sum_{j=1}^{p} Q_{j} \frac{d Q_{j}}{d t} \\
& =2 \sum_{j=1}^{p} Q_{j}\left(\bar{a}_{j}-\sigma_{j}(t)\right) \\
& =2 \min _{\sigma \in<\mathcal{S}>} \sum_{j=1}^{p} Q_{j}\left(\bar{a}_{j}-\sigma_{j}\right) \\
& =2 Q^{\Sigma} \min _{\sigma \in<\mathcal{S}>} \sum_{j=1}^{p} P_{j}\left(\bar{a}_{j}-\sigma_{j}\right) \\
& \leq 2 Q^{\Sigma} \max _{P \in \mathcal{P}} \min _{\sigma \in<\mathcal{S}>} \sum_{j=1}^{p} P_{j}\left(\bar{a}_{j}-\sigma_{j}\right) \\
& =2 Q^{\Sigma} \min _{\sigma \in<\mathcal{S}>} \max _{P \in \mathcal{P}} \sum_{j=1}^{p} P_{j}\left(\bar{a}_{j}-\sigma_{j}\right) \\
& =2 Q^{\Sigma} \min _{\sigma \in<\mathcal{S}>j=1, \ldots, p}\left(\bar{a}_{j}-\sigma_{j}\right) \\
& \leq-2 Q^{\Sigma} \in
\end{aligned}
$$

From this we see that so long as the total queue size is positive then the (euclidean) distance of the queue size vector is decreasing, and so must decrease to zero.

The formal proof of Theorem 3 is given in the appendix. We note that the proof for MaxWeight has several connections with the proof of the Blackwell Approachability Theorem:

1. The argument is constructive and applies a quadratic Lyapunov function.

2. The proof applies the Minimax Theorem. ${ }^{3}$

3. The divergence of the policy follows from a separating hyperplane argument.

All of these properties indicate a close connection between MaxWeight and Blackwell Approachability, which we develop next.

\footnotetext{
${ }^{2}$ Note here we are ignoring arrival rates on the boundary $\partial\langle\mathcal{S}\rangle=\langle\mathcal{S}\rangle \mid\langle\mathcal{S}\rangle^{\circ}$

${ }^{3}$ We note that we apply the Minimax Theorem in the way that we construct our proof but this is not necessarily standard.
} 


\subsubsection{MaxWeight and Approachability.}

Here we show that MaxWeight is an instance of Blackwell's policy, (6), for a specific choice of $R$. Thus a reason for the robust stability properties of MaxWeight can be seen as a consequence of robustness to adversarial perturbations found in Blackwell's Approachability Theorem.

Proposition 1. The MaxWeight policy (14) is an instance of Blackwell's policy (6) and as a consequence of Blackwell's Approachability Theorem it follows that for any subcritical arrival rate $\bar{a} \in<\mathcal{S}>^{\circ}$

$$
\mathbb{E}\left[\frac{1}{T} \sum_{j=1}^{q} Q_{j}(T)\right] \underset{T \rightarrow \infty}{\longrightarrow} 0 .
$$

Proof. For the proof, we construct a payoff matrices $R^{k}, k=1, \ldots, q$ was well as decisions $d^{\prime}(t)$ and adversary decisions $a^{\prime}(t)$ that give queueing behavior. We then show that for these choices the policy of Blackwell corresponds to the MaxWeight policy.

We define the $2 q$-dimensional vectors

$$
\begin{aligned}
& a^{\prime}(t)=\left(a_{1}(t), \ldots, a_{q}(t), 1, \ldots, 1\right) \\
& d^{\prime}(t)=\left(1, \ldots, 1, d_{1}(t), \ldots, d_{q}(t)\right)
\end{aligned}
$$

where we assume that $a(t)=\left(a_{1}(t), \ldots, a_{q}(t)\right)$ is a random variable with mean $\bar{a}(t)$ belonging to $\langle\mathcal{S}\rangle$ and we assume $d(t)=\left(d_{1}(t), \ldots, d_{q}(t)\right)$ belongs to $\mathcal{S}$. For each $k=1, \ldots, q$, we define the $2 q \times 2 q$ matrix

$$
R^{k}=\left(\begin{array}{cc}
\delta_{k} & 0 \\
0 & -\delta_{k}
\end{array}\right)
$$

where $\delta_{k}$ is the $q \times q$ diagonal matrix with only one non-zero diagonal entry in the $k$-th entry which is set equal to one. Notice that

$$
d^{\prime}(t) R^{k} a^{\prime}(t)=a_{k}(t)-d_{k}(t),
$$

for $k=1, \ldots, q$. Thus using the notation of (1), we have that

$$
R\left(d^{\prime}(t), a^{\prime}(t)\right)=a(t)-d(t) .
$$

So, the vector payoff $R\left(d^{\prime}(t), a^{\prime}(t)\right)$ is exactly the change in queue length given in (9). So $\bar{Q}(T)$ as given in (2) is the time averaged queue size

$$
\bar{Q}(T)=\frac{1}{T}\left[Q(0)+\sum_{t=1}^{T}\{a(t)-d(t)\}\right]=\frac{Q(T)}{T} .
$$

This shows that we can consider a switched queueing network within the framework of Blackwell.

We next consider the approachability properties of the switched queueing network. Specifically we consider approachability of the set

$$
\mathcal{Z}=\left\{z \in \mathbb{R}^{q}: z_{k} \leq 0, \quad \forall k=1, \ldots, q\right\} .
$$

Clearly the projection onto this set, $P(t)$, equals zero for any $\bar{Q}(t) \geq 0$. So the hyperplane $\mathcal{H}(t),(5)$, has normal vector $\hat{n}(t)=\bar{Q}(t)$ and so is given by

$$
\mathcal{H}(t)=\left\{r \in \mathbb{R}^{q}: \bar{Q} \cdot r \leq 0\right\} .
$$

Recall the from Blackwell's policy approachability holds when

$$
R(d(t), a) \in \mathcal{H}(t), \quad \forall a \in<\mathcal{S}>.
$$

Thus given the definition of $\mathcal{H}(t)$ and $R\left(d^{\prime}, a^{\prime}\right)$, above, we arrive at the condition

$$
\bar{Q}(t) \cdot d(t) \geq \bar{Q}(t) \cdot a, \quad \forall a \in<\mathcal{S}>,
$$

or equivalently (maximizing over $a \in<\mathcal{S}>$ and noting that $\bar{Q}(t)=Q(t) / t$ )

$$
Q(t) \cdot d(t) \geq \max _{a \in<\mathcal{S}>} Q(t) \cdot a=\max _{\sigma \in \mathcal{S}} \sum_{j=1}^{q} Q_{j}(t) \sigma_{j}
$$


(The equality above holds since a maximizer of a linear function over a polytope must lie on an (extreme) point in $\mathcal{S}$.) Thus by choosing $d(t)$ according to the MaxWeight policy, we maximize the lefthand side which in turn gives us a policy that satisfies the criteria of Blackwell's Approachability Theorem, and thus as a direct consequeunce we have that

$$
\|\bar{Q}(T)\|_{L_{2}} \underset{T \rightarrow \infty}{\longrightarrow} 0
$$

since $\|\bar{Q}(T)\|_{L_{1}} \leq\|\bar{Q}(T)\|_{L_{2}}$, the result (13) holds.

A few remarks about the above result can be made.

1. The rate of convergence can be found to be of the order $O(1 / \sqrt{T})$. Thus the result can be seen as a form of "fluid stability" or "rate stability" for the policy. Because the rate of departures from the network is of the same order as the rate of arrivals. Provided $a \in\langle\mathcal{S}\rangle^{0}$ the result can be improved to form a bound that is $O(1 / T)$.

2. The result proven above holds when the arrival rates can change over time (in an adversarial way) so long as the expected number of arrivals at each time belongs to the interior of the stability region $<\mathcal{S}>^{\circ}$.

3. We note that there are variants of Blackwell Approachability that expand upon the basic version to incorporate general Lyapunov functions (cf. Cesa-Bianchi and Lugosi (2006)). If we apply these alternative algorithms for queueing network scheduling then we arrive the $f$-MaxWeight policies. This is a variant of MaxWeight where the queue size $Q_{j}$ in the MaxWeight policy is replaced by $f\left(Q_{j}\right)$ for a suitably positive increasing function $f$.

4. The argument given in Proposition 1, can be extended to the BackPressure policies. The BackPressure policy is an extension of the MaxWeight policy introduced by Tassiulas and Ephremides (1990). BackPressure allows for routing decisions that send jobs through multiple queues. BackPressure can also be seen to be an instance of Blackwell Approachability for a suitable choice of $R$.

5. MaxWeight as stated assumes unit sized jobs and there is no randomness in the service received by a job. However, the policy can be extended to jobs which have a random size or a random probability of being served. In this case, maximal stability does not hold under the policy (14). We need to consider a policy of the following form

$$
d(t)=\underset{\sigma \in \mathcal{S}}{\operatorname{argmax}} \sum_{j=1}^{q} \mu_{j} Q_{j}(t) \sigma_{j},
$$

where $\mu_{j}^{-1}$ is the mean size of jobs at queue $j$. This can be seen as a consequence of the form of the function $R(d, a)$ in this case. Here service must be given within each matrix $R^{k}$ in order to represent this model within the Blackwell framework, specifically,

$$
\mathbb{E}\left[R^{k}\right]=\left(\begin{array}{cc}
\delta_{k} & 0 \\
0 & -\mu_{k} \delta_{k}
\end{array}\right) .
$$

However arrivals can be implemented as vector applied to these matrices. So arrivals can be specified by an adversary but service must be specified as a parameter of the game. For this reason, the MaxWeight policy is robust to adversarial perturbations in arrivals, but may not have the same property when we consider adversarial job sizes. Nonetheless the mean job sizes and other statistics can still be learned. We discuss this in Section 3.

\subsection{Literature Review}

As mentioned earlier, the original Approachability Theorem is due to Blackwell (1956). The connection with sub-linear regret is described by Blackwell (1954). The use of Blackwell's condition is now commonly applied to analyze the regret of online learning algorithms. See Cesa-Bianchi and Lugosi (2006) for an overview. The text of Cesa-Bianchi and Lugosi (2006) provides a generalized Lyapunov version of Blackwell's condition, which as discussed specializes to well known variants of $f$-MaxWeight in the case of switched queueing networks, see Shah et al. (2012). It has also been shown that sub-linear regret is equivalent to Blackwell approachability, Abernethy et al. (2011). 
The BackPressure policy, of which MaxWeight is a special case, was first introduced by Tassiulas and Ephremides (1990). During this period several commonly used queueing policies were found to be unstable when each queue was nominally underloaded, for instance see Rybko and Stolyar (1992). The BackPressure policy is a popular algorithm that circumvents these issues. It was later found that input-queued switches, which are the routers used in the core of the internet, could have a sub-optimal stability region under the greedy work-conserving strategies used at the time. For this reason the MaxWeight algorithm was proposed by McKeown et al. (1999) as a strategy to achieve maximal stability. Since then the MaxWeight policy has been applied extensively in the analysis of input queued switches. More generally the MaxWeight and BackPressure policies have been widely analyzed in a broad set of queueing models. The paper Dai and Lin (2005) gives an account of their broad applicability in a wide variety of contexts including manufacturing, call centres as well as communications systems. We refer to Georgiadis et al. (2006) for a survey on MaxWeight and BackPressure. Also the recent text Dai and Harrison (2020) provides an excellent introduction to stochastic networks, their stability as well as providing a good account of the various use cases of MaxWeight and BackPressure policy.

The connection between Blackwell Approachability and MaxWeight/Backpressure appears to be new but we note it is a natural consequence of the two approaches. Specifically, the results of Neely (2010b) are particularly relevant to our analysis as it is shown that MaxWeight and Backpressure are robust to changing arrival rates so long as the mean arrival rate lies within the stability set over each time period (see also Neely (2010a)). The literature on adversarial queueing models is also relevant (Borodin et al. 1996). Similar fluid stability results are sufficient for various adversarial queueing models (Gamarnik 1998). MaxWeight has been analysed in the context of adversarial queueing by Lim et al. (2013).

From a theoretical perspective, understanding of MaxWeight has improved through a series of papers analyzing state-space collapse of MaxWeight in heavy traffic, see for instance Stolyar et al. (2004) and Maguluri and Srikant (2016). Also it can was proven that MaxWeight does not enjoy the same robust stability properties that BackPressure enjoys in large networks Bramson et al. (2019). An intriguing fluctuation bound for MaxWeight is found in Sharifnassab et al. (2020). More recent literature on MaxWeight and BackPressure in queueing networks has focused closed systems, such as using it as a decision rule in ride sharing platforms (see Banerjee et al. (2018) and Kanoria and Qian (2019)). Another application concerns road traffic signal control (Le et al. 2015, Varaiya 2013, Xiao et al. 2015), which we will discuss in some more detail in Section 5.

A variety of recent papers combine elements of learning and MaxWeight. One area of research employs MaxWeight-like policies in designing learning and information processing systems, such as those encountered in crowd-sourcing, in order to achieve maximum throughput or minimize regret (Hsu et al. 2021, Massoulié and Xu 2018, Shah et al. 2020b). The novelty here is that instead of working with queues of jobs, the decision maker is trying to minimize certain information backlog that corresponds to "uncertainty" in the system. Another line of work investigates how to augment MaxWeight with explicit learning and side information to improve performance (Krishnasamy et al. 2018, Neely et al. 2012). However, they tend to focus more on parameter estimation, rather than approachabilitiy proporties for the algorithm. We will focus on such methods in the next section.

\section{Regret Bounds and Queueing}

When we analyzed MaxWeight, we saw that stability is achievable under an unknown arrival rate. However, we do require knowledge of service rates to make decisions. So if service rates are not known in advance then they must be learned. Recently there have been a number of papers that investigate sequential learning of service rates in queueing systems. These papers consider how bounds on regret (as defined above) relate to the performance of a queueing system. We will review these shortly, but first we discuss the main ideas by developing a simple example.

\subsection{Regret and service in a queue.}

We suppose that jobs arrive to a single server queue. We let $a_{t}, t \in \mathbb{Z}_{+}$, be the inter-arrival time between $t$-th arrival and the $(t+1)$-th arrival. When we serve a job, we suppose that there are different modes of service that we can select. This represents the manner in which we are going to serve the job, with some service modes being more suitable than others. We let $\mathcal{M}$ be the set of service modes. A policy $\pi=\left(\pi_{t}: t \in \mathbb{Z}_{+}\right)$choses a service mode for each job, i.e. for the $t$-th job at the queue we select service mode $\pi_{t} \in \mathcal{M}$. We let $\tau_{t}^{m}$ be the time spend serving the $t$-th job under service mode $m$. We assume that for each job, one service mode (which is not known to us) will lead to a fast service time, 
$\tau^{\star}=\min _{m \in \mathcal{M}} \tau_{t}^{m}$, and the other service modes will lead to a slower service time. We let $\tau_{t}^{\pi}$ be the time taken to serve the $t$-th job under policy $\pi$. Shortly, in Section 3.2, we will discuss a specific model that relates the service modes to the speed of service. See Figure 4 for a simple example of model just described.

Following our earlier definition, (7), the regret of policy $\pi$ is

$$
\mathcal{R} g(T)=\sum_{t=1}^{T}\left(\tau_{t}^{\pi}-\tau^{\star}\right) .
$$

I.e., the regret is the difference between the total service we receive under policy $\pi$ and the service that we receive under optimal service. Given the inter-arrival times, $a_{t}$, and service times, $\tau_{t}^{\pi}$, the waiting time of the $t$-th customer satisfies the famous Lindley recursion:

$$
W_{t}^{\pi}=\max \left\{0, W_{t-1}^{\pi}+\tau_{t-1}^{\pi}-a_{t-1}\right\} .
$$

The waiting time under the optimal policy is then

$$
W_{t}^{\star}=\max \left\{0, W_{t-1}^{\star}+\tau^{\star}-a_{t-1}\right\} .
$$

In both cases we assume that the queues are initially empty: $W_{0}^{\pi}=W_{0}^{\star}=0$.

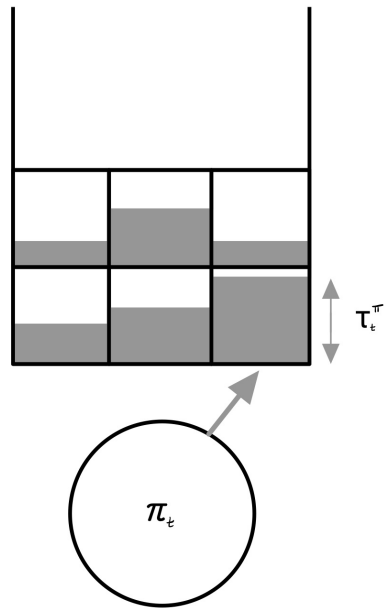

Figure 4: Regret at a queue. Here is a queue with two jobs and three modes of service. The current policy will serve the first job in under the 3rd service mode. This decision has a long service time. We can see that, in hindsight, the 1st service mode would have been best.

The following simple lemma provides a way to compare the waiting time of jobs in terms of the regret of the policy $\pi$

Lemma 1. If $t_{0}$ is the last time before $t$ where the queue for policy $\pi$ is empty, i.e. $W_{t_{0}}^{\pi}=0$, then

$$
W_{t+1}^{\pi}-W_{t+1}^{\star} \leq \mathcal{R} g(t)-\mathcal{R} g\left(t_{0}-1\right)
$$

With this result in hand, there are a host of ways that we can apply regret analysis to a queueing model. In informal terms, here are two ways that one can proceed. First, notice that if $t_{0}=o(t)$, i.e. queues do not empty often, then the term $\mathcal{R} g(t)$ will dominate the above expression, (15). Thus we have a form of rate stability. Specifically

$$
\text { if } \quad W_{t}^{\star}=o(t) \text { then } W_{t}^{\pi}=o(t) .
$$


So if there is some policy under which queues do not grow linearly, then the queues will not grow linearly under $\pi$, either. This is essentially the observation made by Walton (2014). This is the form of the result that one can expect in an adversarial setting. Second, in a stochastic setting we can expect a queue to be positive recurrent, that is $t-t_{0}=O(1)$. Thus the bound is of the form of the expected change in $\mathcal{R} g(t)-\mathcal{R} g\left(t_{0}-1\right)$. In a stochastic multi-arm bandit problem, the regret is often the order $\log t$, So $\log t-\log \left(t_{0}-1\right) \sim \frac{t-t_{0}}{t}$, and we can expect that

$$
\text { if } \quad t-t_{0}=O(1) \text { and } \mathcal{R} g(t) \sim \log t \text { then } W_{t+1}^{\star}=O\left(\frac{1}{t}\right) .
$$

This is essentially the observation made by Krishnasamy et al. (2021) for a specific algorithm, although the same result likely holds in more generality.

As the observations (16) and (16) suggest, there are a host of algorithms that are likely to permit exact analysis; however, one classical learning algorithm that permits a particularly tractable analysis is the perceptron algorithm, which we discuss next.

\subsection{Analysis with the Perceptron Algorithm}

We consider the setting where there are two modes of service $\mathcal{M}=\{-1,+1\}$. With each job, $t$, we associate a context $x_{t}$ and a response $y_{t}$. We assume $x_{t}$ is a bounded vector in $\mathbb{R}^{p}$, i.e. $\left\|x_{t}\right\| \leq D$ for some $D$, and we assume that $y_{t}$ belongs to $\mathcal{M}=\{-1,+1\}$. The context summarizes information about the job and with this information we are allowed to make a service mode decision, $\pi_{t} \in \mathcal{M}$. We assume that the sign of $y_{t}$ determines whether the job receives fast or slow service. Specifically, if the sign of $\pi_{t}$ and $y_{t}$ match then the job receives fast service and otherwise it receives a slower rate of service. That is we assume that

$$
\tau_{t}^{\pi}= \begin{cases}\tau^{\star}, & \text { if } \pi_{t}=y_{t}, \\ \tau^{0}, & \text { if } \pi_{t} \neq y_{t} .\end{cases}
$$

Here $\tau^{0}$ corresponds to the slow service time and so $\tau^{0}>\tau^{\star}$. This correctly classifies the context of a job and leads to faster service.

We restrict ourselves to policies that apply weights to each context in-order to make a decision. Specifically, for weights $w \in \mathbb{R}^{p}$, the policy $\pi_{w}$ with context $x$ makes the decision

$$
\pi_{w}(x)= \begin{cases}+1, & \text { if }\langle w, x\rangle \geq 0 \\ -1, & \text { if }\langle w, x\rangle<0 .\end{cases}
$$

For weights $w$, the loss of the $t$-th job is

$$
\hat{l}_{t}(w)=\mathbb{I}\left[\pi_{w}\left(x_{t}\right) \neq y_{t}\right]
$$

We can upper-bound this by the hinge loss

$$
l_{t}(w)=\max \left\{1-y_{t}\left\langle w, x_{t}\right\rangle, 0\right\} .
$$

It is not hard to check that $\hat{l}_{t}(w) \leq l_{t}(w)$. The resulting optimization

$$
\min _{w \in \mathbb{R}^{p}} \sum_{t=1}^{T} \max \left\{1-y_{t}\left\langle w, x_{t}\right\rangle, 0\right\}
$$

is known as a support vector machine. ${ }^{4}$ Note that

$$
\nabla_{w} l_{t}= \begin{cases}-y_{t} x_{t} & y_{t}\left\langle w, x_{t}\right\rangle<1 \\ 0 & \text { otherwise }\end{cases}
$$

If we apply an online gradient descent algorithm, we get the following update algorithm for the weights $w_{t}$ :

$$
w_{t+1}=w_{t}+\alpha y_{t} x_{t} \mathbb{I}\left[y_{t}\left\langle w_{t}, x_{t}\right\rangle<1\right]
$$

where here $\alpha>0$ is the learning rate of the algorithm and we take $w_{0}=0$. The above algorithm is known as the perceptron algorithm.

Allowing the learning rate to depend on time, the following is a standard bound in online convex optimization (OCO):

\footnotetext{
${ }^{4}$ Often an additional regularization term is added to this objective though we do not consider that here.
} 
Proposition 2 (OCO bound). For any function $\nabla l_{t}(w)$ and $w_{t}$, as above, then for all $w \in \mathbb{R}^{p}$

$$
\sum_{t=1}^{T} l_{t}\left(w_{t}\right)-\sum_{t=1}^{T} l_{t}(w) \leq \frac{\left\|w_{1}-w\right\|}{\alpha_{1}}+\sum_{t=1}^{T} \frac{\left\|w_{t}-w\right\|^{2}}{2}\left(\frac{1}{\alpha_{t}}-\frac{1}{\alpha_{t+1}}\right)+\sum_{t=1}^{T} \frac{\alpha_{t}}{2}\left\|\nabla l_{t}\left(w_{t}\right)\right\|^{2} .
$$

The above result bounds the performance of the sequence of weights $w_{t}$ in comparison with any fixed choice $w$. Thus the above bound is a regret bound. Appropriate conditions on the boundedness of $w_{t}$ and the magnitude lead to sharp regret bounds. A more general overview is given by Hazan (2019).

We pursue this now in the context of the Perceptron Algorithm. We consider the Perceptron Algorithm with a fixed learning rate, $\alpha$, we note that since $w_{0}=0$. The sequence of weights $w_{t}$ are proportional over each choice of $\alpha$. A consequence of the previous bound and this observation is the following classical result:

Theorem 4 (Perceptron Mistake Bound). If there exists $w^{\star} \in \mathbb{R}^{p}$ such that $y^{(t)}\left\langle x^{(t)}, w^{\star}\right\rangle \geq 1$ then the perceptron algorithm is such that

$$
\sum_{t=1}^{\infty} \hat{l}_{t}\left(w_{t}\right) \leq D^{2}\left\|w^{\star}\right\|^{2}
$$

If we apply the perceptron algorithm to the service of rate of customers as considered in Lemma 1 then, since the number of mistakes made by the algorithm is finite, the difference in the regret of the algorithm is bounded by a constant. Thus applying Lemma 1, we arrive at the following result.

Corollary 1. For weights determined by the perceptron algorithm then the waiting time of customers at the queue is

$$
W_{t+1}^{\pi}-W_{t+1}^{\star} \leq D^{2}\left\|w^{\star}\right\|^{2} .
$$

Moreover if $t^{\prime}$ is the first time that $W_{t^{\prime}}^{\pi}=0$ after the last mistake of the perceptron algorithm, then for all $t \geq t^{\prime}$

$$
W_{t}^{\pi}=W_{t}^{\star}
$$

The analysis here is particularly clean as the number of mistakes made by the perceptron algorithm in this "well-separated" setting is finite. ${ }^{5}$ In general the difference in aggregate loss (or number of mistakes) will continue to increase, and the change in losses with decrease rather than be zero after some point. In this case, slightly more careful bounds need to be applied to gain similar results, although the main idea remains the same.

\subsection{Literature}

The application of Lindley's recursion to the regret analysis of a queueing system follows the discussion Walton (2014). Here a result similar to Lemma 1 is derived and the regret for a variety of expert and bandit algorithms is considered. The results of Walton (2014) are primarily concerned with the adversial arrival and service types. The regret of stochastic bandit problems are considered in Krishnasamy et al. (2016). Here a specific algorithm called $Q$-UCB is considered and a detailed analysis of the transition from instability to stable behavior is considered. The discussion in display (17) is a heuristic derivation of this result in Krishnasamy et al. (2016) and suggests that the results hold more broadly than for the $Q$ UCB algorithm. The work is developed further by the authors to consider multiple queues and matching constraints (Krishnasamy et al. 2021). The adversarial setting is considered for a MaxWeight model by Liang and Modiano (2018a). Further formulations for wireless networks (Stahlbuhk et al. 2019) and for network utility maximization are considered (Liang and Modiano 2018b). A strategic adversarial model of queueing is considered by Gaitonde and Tardos (2020a). Here the loss in capacity through adversarial coordination is the object of interest. The authors improve these results exploiting sub-modularity structure Gaitonde and Tardos (2020b).

The set of papers above are amongst the first papers to consider regret analysis within a queueing system. Nonetheless it should be noted that there is an extensive prior literature adressing related problems. For a discounted reward objective the Gittin's index is known to be a Bayesian optimal bandit algorithm, see (Lattimore and Szepesvári 2020, Section 35). There are several works which develop a queueing approach to the multi-arm bandit problem. For instance see Aalto et al. (2009), Jacko (2010),

\footnotetext{
${ }^{5}$ Here well-separates means that there exists $w^{\star} \in \mathbb{R}^{p}$ such that $y^{(t)}\left\langle x^{(t)}, w^{\star}\right\rangle \geq 1$.
} 
Scully et al. (2020) as well as the text Gittins et al. (2011). Further adversarial models of queueing systems are considered in some detail initiated by the work Borodin et al. (1996).

The perceptron algorithm is a classical algorithm considered in machine learning (Novikoff 1963). The mistake bound forms the basis for VC and Radamacher complexity bounds (Vapnik 2013). Online convex optimization and perceptron mistake bounds follow the proofs given in Shalev-Shwartz et al. (2011).

Further, it is worth noting that the analysis of regret only applied to algorithms applied in a online setting where parameters must be fitted statistically. I.e. a regret analysis is not relevant to models trained by simulation. Here only the quality of the final solution reached by the learning algorithm matters. If an algorithm is trained online then care must be taken to the potential changing dynamics and statistics of the environment. Such a view point is taken by Besbes et al. (2015) in the setting of stochastic approximation and by Daniely et al. (2015) in the non-stochastic case.

In practical applications, the effectiveness of an online learning algorithm applied to a queueing system may well depend on the application area. A learning algorithm will always take time to converge to the best decision on average. So the average decision must be a metric of interest and the algorithm must have time to converge. For instance, demand from TCP flows at an internet router will look to overload the router thus the adversarial framework considered in Section 2 would be more appropriate. However, more slowly varying applications such as ride-sharing and matching markets might be more appropriate to the framework described here. Here statistically well-defined customer classes can be learning and optimized over time. This approach is considered by Hsu et al. (2021) where the unknown payoff from service at a queueing system must be learned and maximized subject to servers capacity constraints. Johari et al. (2021) considers a related line of work where server classes must be learned. The work of Liu et al. (2020) consider an online dispatch problem as a sequential learning problem and combine an online learning analysis with virtual queueing ideas in order to manage system capacity constraints. A further area that combines inventory control and online learning is online stochastic bin packing. Here an unknown distribution must be sequentially learned and packed in to unit sized bins. We refer the reader to Gupta and Radovanović (2020) and references therein.

\section{Role of Information}

The algorithms introduced the previous sections generally have a "learning" flavor: the algorithm either explicitly gathers information about unknown quantities in the environment, such as in the case of bandits, or implicitly controls the system in a manner that mitigates the lack of knowledge of such information, such as Max-Weight being able to stabilize the queues despite not knowing the arrival rate. What exactly, then, is the information that is being learned, and how does such information shape the performance and algorithmic design? These questions will guide our discussion in the next two subsections.

At a high-level, by information we are referring to knowledge of uncertain values in the system. As the exposition of Lu et al. (2021) discusses, it is instructive to further consider the following two categories of uncertainty:

1. Epistemic information: this corresponds to static uncertain parameters and system model specifications. For instance, in a typical queueing network, uncertainty in the arrival rate or mean service time belongs to this categories.

2. Aleaoric information: this corresponds to uncertain realizations and stochastic events that manifest over time, and in particular, is unknown even if we have a complete specification of the system model. For example, while we may know that the arrival counts in time step $t$ follows a Poisson distribution with mean 1 (epistemic information), the exact realization of the number of arrivals $A_{t}$ is still uncertain. Knowledge of the realized value of $A_{t}$ would correspond to aleaoric information.

For the purpose of our discussion, one can roughly think of epistemic information as about static quantities, whereas aleaoric information captures dyanmic realizations of randomness over time.

Aleaoric information: past, present and future We will focus in this section on the impact of aleaoric information. We will have a broader discussion about the role of epistemic information in the next section in the context of reinforcement learning. Note that, the bandit-inspired algorithms and the Max-Weight algorithm both focus on addressing epistemic uncertainty.

We will further orient our discussion along three sub-type of aleaoric information, determined by where the information is situated along the time axis, relative to the present time frame: 
1. Information about the future inputs, a.k.a. predictive information. E.g., how many jobs will arrive and to what queues in the next 5 hours.

2. Information about the current system state, such as the queue lengths at various servers in a load-balancing model.

3. Information about the past, possibly stored as part of the algorithm's internal memory.

Measuring the value of information One of the core questions when it comes to the role of information is its decision-theoretic value, expressed as the performance improvement that accessing such information may enable. For instance, we may seek to find a function $v$ such that, given $x$ amount of future information, we may claim to achieve $v(x)$ amount of performance improvement.

Two immediate questions come to mind. First, in various stochastic resource allocation problems, what how does the value $v(x)$ depend on the amount of information, $x$ ? In a centralized decision-making framework (e.g., barring competing selfish agents), more information cannot hurt the decision maker, so we would naturally expect $v(x)$ to be always non-negative and monotone non-decreasing. A more interesting question can be: how fast does $v(x)$ increase as the amount of information $x$ increases, if at all. As we will see in some of the results surveyed in this section, sometimes a seemingly small amount of information can lead to significant performance improvement. Conversely, performance improvement could quickly plateau after obtaining "enough" information, beyond which more information only brings small, marginal benefits.

Once we understand how much benefit information can bring, an immediate follow up question would be how to achieve the optimal performance improvement $v(x)$ using efficient algorithms. A notion that is suitable for most engineering applications is to require that the resulting decision policy to be computationally tractable. A taller order might be warranted when the application involves humans, such as in a call center or health-care setting, we would ideally like the algorithm to be simple and intuitive from the perspective of a human operator.

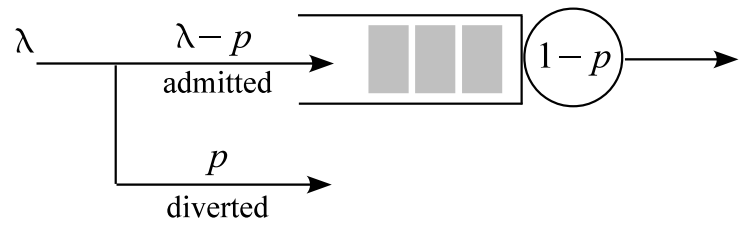

Figure 5: A queueing admission control model with future information (Spencer et al. 2014).

\subsection{Improving Queueing with Future Information}

Our first example concerns admission control in a queueing system using future information (Spencer et al. 2014). We will look at an operator who may access to a third-party oracle (via predictive algorithms, machine learning, or simply a side-channel) that offers a look-ahead window containing future arrival and departure patterns. We will see that in this example, not only does queueing delay improve with future information, but the function $v(x)$ experiences a sharp increase early on, before petering out, suggesting that the system can benefit a lot from having access to a moderate amount of information.

The model is depicted in Figure 5. An single stream of jobs arrive to a queue following a Poisson process with rate $\lambda \in(0,1)$. Service variability is modeled by an independent Poisson process of rate $1-p$ : suppose that there is a jump in this process at time $t$, then the queue is reduced by 1 if it is not empty, or stays at zero, otherwise. It is easy to show that in this system the queue length process, $Q(t)$, would evolve according to the same law as the number of jobs in system (including in service) in a conventional $M / M / 1$ queue, with arrival rate $\lambda$, service rate 1 , and mean job size $1 /(1-p)$.

Now, notice that if the arrival rate $\lambda$ exceeds the service rate $1-p$ the queue length will eventually grow to infinity. We would thus assume that the policy designer is to make some admission decisions on a dynamic basis: for each arriving job, the algorithm is to decide whether the job is to be admitted to the queue, or diverted, in which case the job leaves the system directly. Because diversions are costly (the diverted job is either not receiving the needed service, or needs to be served elsewhere), we impose a constraint that the average rate of diversion be at most $p$. This model of admission control has a long history and has been studied in various applications, ranging from Internet congestion control to 
ambulance divergence for Emergency Departments; see references in (Stidham 1985, Spencer et al. 2014, $\mathrm{Xu}$ and Chan 2016).

A key question is how to control admissions in such a way that minimize the waiting time experienced by the admitted jobs, while obeying the diversion rate constraint. Everyday intuition would suggest that jobs should be turned away when the queue is already very long, and conversely, we should probably admit the job if the queue is nearly empty. In other words, we could implement a threshold-policy where a job is admitted if any only if the queue length at the time of arrival is less than a certain threshold, and the threshold would be chosen to be smallest one that results in a feasible admission rate. Showing that this is indeed optimal is non-trivial, and was elegantly carried out in Stidham (1985). In particular, under the optimal policy (which uses a threshold rule), the average steady-state queue length scales as:

$$
\mathbb{E}[Q] \sim \Theta\left(\log \frac{1}{1-\lambda}\right), \quad \text { as } \lambda \rightarrow(1-p)
$$

Here, the limit $\lambda \rightarrow 1-p$ corresponds to the conventional heavy-traffic regime, where the arrival rate of the admitted traffic, $\lambda-p$, approaches the service capacity $1-p$. Note that as $\lambda \rightarrow 1-p$, the queue length explodes to infinity, as one would expect from standard heavy-traffic theory of queues, albeit at a more modest, logarithmic rate compared to the typical $1 /(1-\lambda)$ heavy-traffic delay scaling.

Let us now introduce future information in this model. Suppose now that the decision maker is equipped some advanced knowledge of the future arrivals and potential services in the form of a lookahead window: at any time $t$, we will assume that the algorithm is able to access the realizations of both the arrival and service Poisson processes in the time window $[t, t+L)$. That is, the decision maker not only has at their disposal how long the queue is now, but how arrival and service will materialize in the next $L$ units of time. We would expect that the availability of such future (aleaoric) information should allow the algorithm to plan more proactively and improve performance. But how large of an improvement can we get, and how much future information do we need in order to make such improvement possible?

It turns out that future information does make a big difference. Specifically, it is shown in Spencer et al. (2014) that there exists some universal constant $a>0$, such that if that the size of the lookahead window $L \geq a \log \frac{1}{1-\lambda}$, then the queue length under an optimal policy satisfies:

$$
\mathbb{E}[Q] \rightarrow \frac{1-p}{p}, \quad \text { as } \lambda \rightarrow(1-p)
$$

That is, equipped with a sufficient amount of future information, the heavy-traffic queue length does not diverge, but instead converges to a finite constant, a sharp departure from the scaling in (18).

We have thus seen that having access to some future information could bring potentially massive performance improvement. However, (19) is only half of the picture, as we might want to know whether the same performance improvement can be attained with much less information. The answer turns out to be negative, as demonstrated by the following lowerbound by $\mathrm{Xu}$ (2015): there exists another positive constant, $b, b<a$, such that if the lookahead window size $L \leq b \log \frac{1}{1-\lambda}$, then the queue length under any algorithm must satisfy:

$$
\mathbb{E}[Q] \sim \Omega\left(\log \frac{1}{1-\lambda}\right), \quad \text { as } \lambda \rightarrow(1-p) .
$$

Comparing the above with (18) and (19), we see that, quite surprisingly, the performance deteriorates to the same level as an online algorithm, as soon as the lookahead window size $L$ drops by even a constant factor, $a / b$. In other words, $\log \frac{1}{1-\lambda}$ appears to capture a critical threshold for performance-relevant future information, where queueing delay is highly sensitive with respect to whether $L$ is greater or less than this level.

The above results also reveal an interesting duality between information and stead-state queue length. While one may expect future information to help reduce the steady-state queue length, it may be surprising that they are related to each other so strongly as to be almost interchangeable. Examining (18) through (20) shows that the system can essentially operate in one of two regimes, as the system load $\lambda$ approaches 1:

1. Information-rich: if the lookahead window size grows faster than $\Theta\left(\log \frac{1}{1-\lambda}\right)$, then the steady-state queue length would be bounded,

2. Information-scarce: if the lookahead window grows slower than $\Theta\left(\log \frac{1}{1-\lambda}\right)$, then the steady-state queue length would grow at rate $\Theta\left(\log \frac{1}{1-\lambda}\right)$.

That is, the operate "pays" a cost of $\Theta\left(\log \frac{1}{1-\lambda}\right)$ either in information or queue length. 
Insight on Policy Design We may also be interested in knowing how policy design should adjust to the availability of future information and forecasts. Do we need to drastically revamp our usual intuition, or are we only looking at minor tweaks to existing policies?

The reality is likely the former. One of the most striking findings of Spencer et al. (2014) is that not only is the optimal control with future information different from the one without, but the difference is so large that it would almost appear counter-intuitive. As discussed earlier, the intuition for an online version of the admission control problem suggests that a good admission policy should follow some threshold rule and admit arrivals to the queue only when the queue is short. In contrast, Spencer et al. (2014) show that when future information is available, the optimal admission policy does almost the opposite: it is more likely reject jobs when the queue is short, and admit when the queue is long. In the extreme case where the lookahead window size is infinite, the optimal policy would only reject jobs when the queue is empty. Why does future information lead to such drastic changes in optimal admission rules?

In some sense, this departure is but an instance of a broader shift from being reactive to proactive as future information becomes part of our decision-making apparatus. In the online setting, since the decision maker has no knowledge of the future realizations of arrivals and services, the build-up in the queue serves as the only warning of congestion. Consequently, the decision maker can react to bursts in arrivals only after they have already occurred. When future information is available, however, the emphasis of the decision maker naturally shifts from what has happened to what will happen, and as such, the current "state" of the system (e.g., queue length) will carry less significance as the amount of future information grows. In the admission control problem, if the decision maker is to foresee future arrivals, then rejecting jobs that occur at the beginning of a burst of arrivals will prove far more profitable than rejecting those jobs that arrive later in the same bursty period. This is because rejecting early arrivals reduces waiting for all subsequent arrivals, whereas rejecting the later arrivals does not have the same "domino effect." It is for this reason that the optimal policy under future information tend to be more aggressive in turning away jobs when the queue is small; not because the queue's being small in itself forebodes future congestion, but simply that early arrivals in a burst of jobs tend to, by definition, arrive when the queue is small. Clearly, with a lack of foresight, it would not have been possible for an online policy to have such discernment.

While these specifics ways in which policy design changes as a result of more future information may or may not generalize to other stochastic systems, a broader point to be made here is that variations in information structure likely will require us to re-evaluate our usual intuition, and sometimes in fairly drastic ways.

\subsection{Communication and Memory in Load Balancing}

Our second example from Gamarnik et al. (2018) shifts the focus of information from the future to that of the present and past. Here, the decision makes may not even have the complete information about the current state of the system. A typical application that motivates such consideration is that of a large data center, where it is often expensive, if not impossible, for a scheduling algorithm to have at its disposal the real-time state information on all servers and queues. In this case, the lack of accurate state information orignates from two possible sources:

1. Limited communication: the decision maker may not be able to communicate frequently with all the queues and servers.

2. Limited memory: there is not enough memory to retain all past information.

To make matters more interesting, it turns out that the effects of communications and memory are often intertwined. With unlimited communication capability (and suitable Markovian assumptions), the true system state is readily accessible and there would be little need for memory. Conversely, when communications are limited, memory becomes crucial as it compensates for the lack of real-time information by piecing together last observations to form a better estimate of the system state. Considering that communication and memory can both be prized resources in large-scale queueing networks, how they jointly impact performance is an important question.

The tension between communication, memory and system performance was elegantly captured by a model proposed by Gamarnik et al. (2018) in the context of load balancing. The system is that of a standard load balancing setup, where a single dispatcher routes jobs, arriving at rate $\lambda n$, to a collection of $n$ parallel servers each operating at unit speed. The dispatcher however does not have access to the queue lengths at all the servers by default, but must rely on messages sent from the servers to gather 


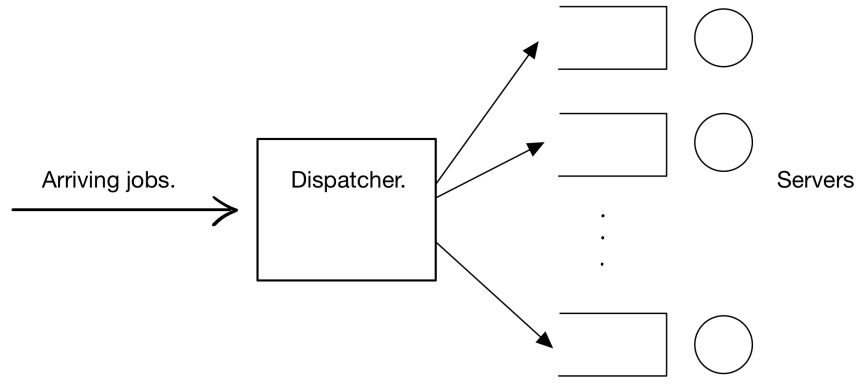

Figure 6: Load balancing with a single dispatcher (Figure 1, Gamarnik et al. (2018)).

such information. A main innovation of this model is its systematic accounting of the communication and memory overhead.

1. Communication model: when a server idles, it sends messages to the dispatcher according to a

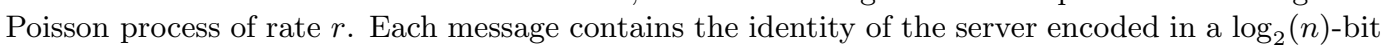
binary string. The server sends nothing if it is currently busy.

2. Memory model: the dispatcher is equipped with a finite-sized memory bank of $c \log _{2}(n)$ bits. Given that it takes $\log n$ bits to specify the index of a single server, this is effectively the same as the dispatcher being able to store the identities of $c$ (idle) servers. Whenever the dispatcher receives a new message, it adds the identity of that server to its memory, and discards it if the memory is already full.

Under the above memory and communication model, Gamarnik et al. (2018) study the delay performance of the following natural routing policy. Upon the arrival of a new job, the dispatcher randomly chooses a server ID from its memory bank, if it is non-empty, and sends the new job to the said server while erasing the ID from the memory. If the memory bank is empty, then the dispatcher simply routes the job to a randomly chosen server. The main result of Gamarnik et al. (2018) characterizes the resulting delay as a function of the level of memory and communication available. In particular, they consider the regime as the number of servers $n$ tends to infinity, and distinguish whether the average system-wide queueing delay vanishes in the limit.

Their main results are summarized in Figure 7. There are several interesting takeaways. First, as one would expect, delay improves as the amount of memory or message rate increase. For instance, in the "High message regime," the messaging rate of each server tends to infinity as $n$ increases. This high rate of message allows the system to achieve vanishing delay even when the dispatcher has only a limited memory size, capable of storing only a bounded number of server IDs ( $c>0$ but bounded). In contrast, for the same memory size, if the messaging rate per server remains bounded, then delay becomes strictly positive in the large-server limit. The same complementarity between memory and communication is partially mirrored in the "High memory regime," where the servers send messages at a constant rate of at least $\lambda$, but the dispatcher can remember an unbounded number of server IDs. Here, the lack of communication is made up for by an abundance of memory, and vanishing delay is again achievable.

A second, and perhaps more striking, insight is that the roles of memory and communications in this model are not entirely symmetrical. In the "High memory regime," the messaging rate per server is assumed to be bounded. In this case, having more memory alone is not sufficient for achieving vanishing delay. In particular, assuming that the number of server IDs the memory can store grows to infinity as $n$ increases $(c \rightarrow \infty)$, then vanishing delay is achieved if and only if the messaging rate per server is at least $\lambda$. Recall that $\lambda$ is exactly the average arrival rate per server. So another interpretation of this result is that the servers need to communicate its idling status at the rate of one message per job in order for more memory to have a meaningful impact on delay performance.

It is worth-noting that the analysis carried out in Gamarnik et al. (2018) applies to the specific dispatching policy. While it is conceivable that some other policy could utilize the same memory and communication resources more efficiently, a follow up paper (Gamarnik et al. 2020) shows that any potential improvement will be limited. They prove that under a certain natural symmetric assumptions, any policy operating in the "Constrained regime" (see Figure 7), where the messaging rate per server stays bounded and the memory size is on the order of $\log n$, necessarily incurs a strictly positive limit delay as $n \rightarrow \infty$. 


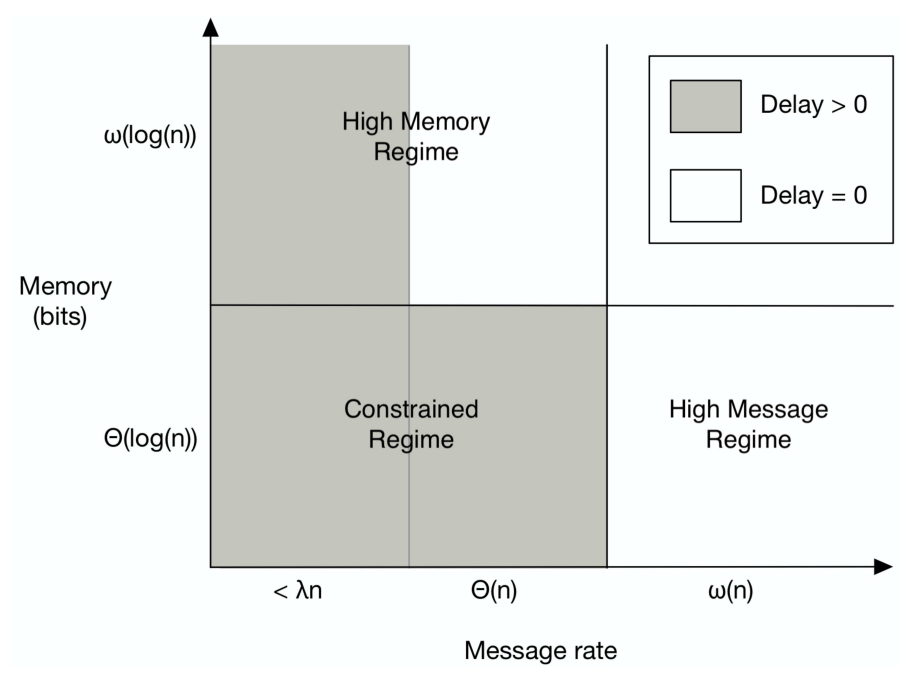

Figure 7: Delay as a function of memory and communication (Figure 3, Gamarnik et al. (2018)).

The interplay between memory, communication and system performance in a queueing network has also been investigated by Xu and Zhong (2020) in a different system model. They study dynamic resource allocation in a single-hop stochastic processing network model under imperfect communication and limited memory. Unlike Gamarnik et al. (2018), where communication is captured by messaging rates, $\mathrm{Xu}$ and Zhong (2020) considers a more general communication model using memoryless Shannon channels in information theory, which would allow for capturing both rate-limited and noisy communication. The focus of $\mathrm{Xu}$ and Zhong (2020) is centered around understanding how to achieve the maximum throughput region when the decision maker can access the state of a queueing system only through a noisy and rate-limited channel, when the algorithms are memory-limited. The main results of Xu and Zhong (2020) consist of a family of Episodic MaxWeight policies that (approximately) achieve the maximum stability region under various combinations of memory and channel conditions. As another departure from the above load-balancing model, in which only the dispatcher possesses a memory, both the queues and scheduler in Xu and Zhong (2020) have their own memory bank. A surprising conclusion from the results in $\mathrm{Xu}$ and Zhong (2020) is that it is not only how much memory that matters, but also where the memory is located: granting more memory to the scheduler, rather than to the queues, leads to a significantly larger impact on the system's stability region.

\subsection{Literature Review and Discussions}

There has been a growing body of literature that examines the impact of predictive information in queueing networks. $\mathrm{Xu}$ and Chan (2016) studies admission control in Emergency Departments where the operator may have access to limited and noisy future information. They propose a family of admission control policies that provably outperforms the optimal online policy at all arrival rates, in contrast to the policy in Spencer et al. (2014) which is only optimal in the heavy-traffic limit as $\lambda \rightarrow 1$. Using simulations based on historical hospital admission data, $\mathrm{Xu}$ and Chan (2016) also demonstrate that leveraging future information can lead to substantial improvements in queueing delay over online policies even if the predictions are noisy and the lookahead window size limited. Ata and Peng (2020) applies a version of the model with future information of Spencer et al. (2014) to the problem of admission control in a call center with call-back options. A key insight there is that the type of proactive admission policies that are shown to be optimal in the over-loaded setting of Spencer et al. (2014) $(\lambda>1-p)$ remains effective in an under-loaded system $(\lambda<1-p)$.

In some cases, having information about future arrivals goes hand-in-hand with being able to intervene on a more proactive basis. Huang et al. (2015) examine proactive scheduling policies that improve the standard BackPressure algorithms in a queueing network. In this model, future arrivals to a queue can be not only predicted, but also served, in advance. In a similar spirit, but different domain, Hu et al. (2020) uses a queueing model to study how to provide proactive care in a healthcare application, where the care provider strives to achieve better treatment outcomes by proactively intervening on a patient who is at the risk of deteriorating in the near future. 
There is a vast body of literature on dispatching policies for load balancing that aim to reduce the communication overhead while maintaining desirable delay performance, including the celebrated powerof- $d$-choices algorithm, where the dispatcher queries $d$ randomly sampled servers and sends the arriving job to the least loaded among them. Mitzenmacher et al. (2002), and more recently Anselmi and Dufour (2020), Hellemans and Van Houdt (2020), study the impact of dispatcher's memory on the performance of this class of algorithms. To the best of our knowledge, Gamarnik et al. (2018) is the first to formally model the joint impact of communication and memory.

\section{Reinforcement Learning in Queues}

We now discuss the challenges in applying Reinforcement Learning theory to Queueing systems. This discussion acts as a literature review of recent progress in the field. We highlight novel approaches in applying reinforcement learning in the context of queueing systems.

We will assume some knowledge of the formalism of Markov decision processes and reinforcement learning, such as $Q$-learning, policy gradients, function approximation, as well as the important role of simulation in training models. To very briefly summarize, a Markov decision process (MDP) is a Markov processes where the evolution of the process is also influenced by actions which are chosen at each time step by a policy. The purpose of the policy is to maximize the expected cumulative rewards received by the process. The optimal policy and its value can be calculated using the Bellman equation. In practice, the evolution of the MDP and its rewards is often unknown and instead must be estimated from data. The suite of algorithms for this joint estimation and optimization is referred to as reinforcement learning (RL). Often the MDP objective has an unreasonably large complexity, thus different forms of function approximation must instead approximate the Bellman equation or directly perform an approximate policy optimization on the MDP objective. Since the early '90, and particularly over the last 5 years, neural networks have been a commonly used framework for function approximation. Successful policies can require large amounts of data to be learned. Thus policy training is often performed via simulation. We refer the uninitiated reader to text such as Sutton and Barto (2018), Bertsekas (2019) and Szepesvári (2010) for introductory accounts.

Below we emphasize the role of queueing models as an important application area for early reinforcement learning models. We then discuss research challenges in the theory of reinforcment learning when applied to queueing systems. We review recent literature that addresses a number of these challenges and finally emphasize their importance in emerging application areas.

\subsection{Queueing in Early RL Literature.}

The application of dynamic programming and reinforcement learning to queueing systems has existed almost since inception. A good example is the work of Crites and Barto (1996). The paper considers allocation of a set of elevators in a building in order to minimize waiting time. The work is amongst the earliest examples of Q-learning with neural networks. The paper highlights a number of specific challenges that can occur within a queueing system: time is continuous and so the times at which decisions are made is a part of the modeling process (in order to regulate the branching factor); there is often incomplete state information (for instance about the number of people waiting); there is decentralized coordination required between different agents; and the stochastic and potentially non-stationary arrival of passengers must be modelled. Many of these issues remain challenges for applying reinforcement learning in queueing systems.

Works of Zhang and Dietterich (1995) consider the application in job shop scheduling problems. Here complex planning constraints are approximated. Singh and Bertsekas (1997) gives an early example of reinforcement learning applied to dynamic channel allocation. Here a channel assignment problem in a cellular telecommunication system is modeled as a dynamic program and a reinforcement learning solution with function approximation is found to outperform the best heuristics. The application of neural network reinforcement learning to inventory management is considered by Van Roy et al. (1997). In addition to neural network approximations, the cross entropy method when first applied to reinforcement learning was applied to inventory control as an example application by Mannor et al. (2003). From these works it is clear that after inception new reinforcement learning methods can readily be applied to a queueing system.

The success of these methods does depend greatly on the predictability of the underlying queueing process. There is a risk of over-optimizing a system for incorrect parameters. As a related anecdotal example, we consider the routing of telephone calls. Here AT\&T would perform periodic learning and 
optimization in order to determine dynamic routing of telephone traffic, see Ash (1995). However, it was found that Dynamic Alternative Routing (DAR) Gibbens et al. (1988), where routing decisions are made based on local blocking decisions, was found to be much more successful. Here, centralized learning and optimization of (epistemic) parameters performed worse than decentralized state dependent (aleatoric) optimization. This is because the local state provided a better representation of the decisions to be made rather than the estimation of the state evolution. The role of current information in decision making, as discussed in the previous section, remains important here.

As we will discuss next, it continues to be the case that queueing systems provide a rich set of models where simulation and reinforcement learning methods are applicable. Nonetheless it is also reasonable to say that even in simple settings mathematical guarantees, correctness and convergence of reinforcement learning algorithms is not well understood. ${ }^{6}$

\subsection{Challenges in applying Reinforcement Learning Theory to Queue- ing Networks.}

There is a growing interest in the application of reinforcement learning to queueing systems, but there are evidently challenges. We need algorithms that learn parameters that we know to be fixed but provide flexibility to model variability. To do this, as the previous section suggests, models require correct and extended notions of state information. For instance information of future arrivals can improve decision making for these transient parameters, or providing contextual information on service rates. However, decentralization, continuous time modeling, incomplete state information, and simulation model misspecification can all inhibit our ability to leverage the benefits of reinforcement learning in a queueing system.

Nonetheless the potential practical power of reinforcement learning methods remains appealing. Many features of a queueing model cannot be well modeled in closed form and so simulation- and data-driven solutions have the ability to capture features that are not well represented by handcrafted models. (For example, it would be better to predict the speed up and slow down of vehicles at a junction rather than create a closed-form mathematical model.) Before discussing recent literature, we highlight challenges that apply to the development of reinforcement learning for queueing systems:

- Unbounded State Space. Queueing systems often have unbounded state spaces, while theoretical results on reinforcement learning assume large but finite state spaces. Function approximation can help alleviate this; however, simulations for small queue sizes may lead to sub-optimal performance for a system in higher-load, which is often the scenario that the system designer cares most about.

- Unbounded State Cost. Even if function approximation may assist, queueing systems can have long regenerative cycles and thus large mixing times. Thus, if we wish to minimize queue size or delay, then the associated costs will be unboundedly large.

- Stability Guarantees. We may wish to guarantee a level of first order performance. We may wish to optimize cumulative rewards subject to stability over a range of loads. There are relatively few guarantees of robustness to parameter uncertainty in a trained reinforcement learning model.

- Non-stationarity. Parameters may change in time. Daily variations can be modeled through the use of contexts. However, in settings such as communications, fluctuations in load can be erratic, which again leads to the risk of learning one part of the state space at the cost of performance out of sample.

- Decentralized Coordination. A queueing systems often consist of interconnected, geographically and logically diverse components. Frequently, centralized coordination is not possible and simulation of the whole system may not be possible either. The optimization of individual components should contribute to the overall optimality of the system as a whole.

- Dependence on Simulation. As with other application areas such as robotics, a simulation driven solution may used to train models but these simulated behaviors might not found in reality. In a road traffic setting, a simulator might incorporate the physical changes of a vehicle but may not account for events such as change lanes or drivers accelerating through an amber light.

- Continuous Time Modeling. Although it is not an unassailable challenge, it is worth noting that many queueing applications are continuous time and thus appropriate decision epochs must be defined. There is a trade off between reducing branching factors while allowing for a suitably rich set of policies to be implemented over time.

\footnotetext{
${ }^{6}$ Beyond canonical assumptions such as finite state space with each state having a lower bound on the proportion of time visited.
} 
- Incomplete State Information. In many settings, the state of a queueing system may not be fully observable or may only be locally observable. In internet congestion in one part of a system may only be sensed locally. In a data center a dispatcher may only be able to gain information on a subset of its servers. We may not know how may people are waiting at a traffic light when a button is pressed.

The above list is by no means exhaustive but covers many issues that RL theory does not currently address for queueing systems.

\subsection{Recent literature.}

Here we review recent theoretical works that look to bridge the gap between theory and practice for reinforcement learning applied to queueing systems.

The paper of Shah et al. (2020a) looks to address the issue of infinite state spaces found in queueing systems. The method considers a setting where their is know to be a Lyapunov function providing stability for some (optimal) policy. The approach pays particular emphasis on evaluating actions for, thus far, unvisited states. Here, the online algorithm does have access to an oracle (or simulator) that can predict future performance from the current state. The paper of Liu et al. (2019) take a different approach by truncating the state-space on which reinforcement learning can be applied. Here it is assumed that there is a known but perhaps sub-optimal policy (such as Max-Weight) that can stabilize the queueing network. A standard RL algorithm is used for learning when the state space is sufficiently small; however, once the Lyapunov function associated with this policy increases beyond some threshold the sub-optimal stabilizing policy is applied.

Although not explicitly analyzing queueing networks, the work of Qu et al. (2020) does much to analyze the effects of decentralized learning and control in a network setting. Here is it proven that if separate nodes of a network implement actions and if the impact of actions at neighboring nodes is delayed, then applying reinforcement learning locally yields a policy that is within a constant factor of the globally optimal policy. The factor in the degradation can be given by either the discount factor of the MDP or by the mixing time of the Markov decision processes.

The previous papers a focus on tabular reinforcement learning methods. However, in practice, function approximation is more commonly applied to yield a solution. The paper of Dai and Gluzman (2020) analyses on the application of deep reinforcement learning to queueing systems. The paper considers the implications of proximal algorithms such as Proximal Policy Optimization and Trust Region Policy Optimization. Here algorithms estimate and optimize in a similar manner to the Policy Iteration algorithm. Dai and Gluzman (2020) focus is on the application to queueing problems with an average cost objective under a moderate to heavy load. The authors present a number of Lyapunov function arguments that modify the policy optimization step of their algorithm. Further they introduce new variance reductions techniques for their value function estimates. These improvements result in pronounced performance benefits for their algorithms in a queueing setting.

In addition to proximal algorithms, policy gradient approaches have also been considered in the context of queueing examples by Zaki et al. (2021b) and Zaki et al. (2021a). Here the task of the MDP formulation is to discover the best mixture amongst a given set of controllers. Under deterministic gradient updates regret bounds are derived.

An under area of investigation is if know formulas can be used to improve the optimization of a queueing system. Albeit not a strictly reinforcement learning setting, the paper of Dieker et al. (2017) should how closed form queueing expressions can be incorporated into stochastic gradient descent procedures in order to expedite the optimization of a queueing network model.

A similar approach exploiting the structure of the queueing system is to simply start with an MDP queueing system with a known solution and then to use this structure along with parameter learning in order to establish theoretical bounds. This approach is taken in the case of inventory control see Agrawal and Jia (2019). Here the base-stock policy is known to be optimal and the value function and cost functions are know to be convex. Through this precise regret bounds are established for an online policy in comparison with the best base-stock solution.

\subsection{Application Areas}

We review a range of queueing application areas where reinforcement learning approaches have been investigated. As discussed applications include healthcare, manufacturing, transportation and communications. The studies below for the most part focus on training reinforcement learning algorithms through simulation. 
Dai and Shi (2019) consider the application of reinforcement learning for the optimization of hospital patient inflows. Here the overflow decision problem is cast as an average cost MDP. An approximate dynamic program is formulated and verified. Emergency response and vehicle assignment approaches to have been applied as MDPs by van Barneveld et al. (2018). Reinforcement learning formulations of this are considered by Lopez et al. (2018). Feng et al. (2020) develop the work of Dai and Gluzman (2020) and apply this to ridesharing systems. See Qin et al. (2019) for a review and discussion on the application of reinforcement learning to Didi's ride-sharing fleet.

Traffic signal control is a further transport applications area. The SurTrac system in the US applies estimation along with a forward dynamic programming solution, see Smith et al. (2013). Companies such as Vivacity explicitly apply deep reinforcement learning in their traffic control system. ${ }^{7}$ See Cabrejas-Egea et al. (2021) for a recent study that demonstrates the ability of deep RL to outperform current commercial traffic signal controllers.

Studies of distributed reinforcement learning for manufacturing processes are considered in Qu et al. (2018). Here the stochastic processing network model of Dai and Harrison (2020) is considered within a reinforcement learning framework. Further studies on machine repair are given by server speed optimization are given by Prabuchandran et al. (2016). Semiconductor manufacturing is investigated by Park et al. (2019).

Applications in communications systems and data centres was initiated by Tesauro et al. (2005b). See also Tesauro et al. (2005a) and Tesauro et al. (2006) for further data centre applications of RL. The area has received further attention with Deepmind's application in Google Data centers. ${ }^{8}$ A more recent study focusing more on congestion control protocols is given by Tessler et al. (2021).

The training reinforcement learning algorithms in large state spaces often requires simulated data. However, there are few standardized simulation environments for queueing systems. For instance, the generic simulators like Open AI's gym environment are required; the ORSuite simulator is a recent simulation environment that looks to bridge this gap, see Archer et al. (2021). Within specific fields there are respected simulation environments like ns3 simulator ${ }^{9}$ for networking, SIMGrid for high performance computing $^{10}$ or Vissim ${ }^{11}$ and $\mathrm{SUMO}^{12}$ for traffic control. Also see Pan et al. (2021) for a recent simulator for hospitals. A hope is that established simulators and problem sets emerge, so that the performance of different polcies can be compared.

Due to their structure some queueing problems still remain challenging for reinforcement learning approaches. For instance the DAG scheduling problem in heterogeneous computing remains challenging due the large array of potential DAGs that a platform must support. Nonetheless, dynamic programming heuristics remain popular, see Topcuoglu et al. (2002). Further, in many practical applications (air traffic control or hospital assignment) there is human level control which governs part of the dynamics and the costs and rewards of the process. Thus a set of solutions are likely required to be readily available. These factors may limit that ability to consider completely model-free learning to be applied some application areas.

Nonetheless a more empirical approach to research on queues and queueing networks will likely stimulate new research. A hope is that queueing theoretical research can be development more closely with the increased acceptance and use of exploratory studies.

\section{Conclusions}

In this tutorial, we have seen how classical queueing algorithms such as MaxWeight and Back Pressure can viewed as an application of Blackwell's Approachability Theorem. Through this we see connections between queueing and online learning theory. This online learning approach does not require the explicit statistical estimation of parameters. However, to consider more general models, we see that the underlying parameters such as service rates must be estimated. We discuss this next and develop connections between statistical learning of sequences of data and the Lindley's recursion for the G/G/1 queue. Through this we develop a notion of regret for a single server queue. As an example we showed how the perceptron algorithm could be used for the classification of customer service at a queue and we bound the impact that this has on the queue length of the policy implemented and the (unknown) optimal policy. We then discuss

\footnotetext{
${ }^{7}$ https://vivacitylabs.com/technology/junction-control/

${ }^{8}$ https://deepmind.com/blog/article/deepmind-ai-reduces-google-data-centre-cooling-bill-40

${ }^{9}$ https://www.nsnam.org/

10 https://simgrid.org

${ }^{11}$ https://www.ptvgroup.com/en/solutions/products/ptv-vissim/

12 https://sumo.dlr.de/docs/index.html
} 
the role of state estimation when compared with parameter estimation. Here we discussed two examples. The first considers the importance of future information in making access control decisions. The second considers the importance of memory in load balancing systems. Finally, we review the application of reinforcement learning highlighting the importance of reinforcement learning to solve practical queueing challenges as well as highlighting current gaps between theoretical results in reinforcement learning and their applicability to modern queueing problems.

Certainly we anticipate that over coming years approaches to information, learning and control will grow in their applicability to queueing networks, and we hope that this tutorial provides a comprehensive introduction and reference to modern methods in this emerging area.

\section{A Appendix}

\section{A.1 Proof of Blackwell's Approachability Theorem}

We now restate and prove Blackwell's Approachability Theorem.

Theorem 5 (Blackwell's Approachability Theorem). The following are equivalent

1. $\mathcal{Z}$ is approachable.

2. For every $q$ there exists $p$ such that $R(p, q) \in \mathcal{Z}$.

3. Every half-space containing $\mathcal{Z}$ is approachable.

Proof. First we need to show that the decision $d(t)$ given in (6) exists. To this end, we recall that the Minimax Theorem states that for a $p \times q$ matrix $M$ it holds that

$$
\max _{a \in \mathcal{A}} \min _{d \in \mathcal{D}} d^{\top} M a=\min _{d \in \mathcal{D}} \max _{a \in \mathcal{A}} d^{\top} M a .
$$

A less compact way of writing the Minimax Theorem is that for every $v \in \mathbb{R}$

$$
\forall a \in \mathcal{A} \exists d \in \mathcal{D} \text { such that } d^{\top} M a \leq v \Longleftrightarrow \exists d \in \mathcal{D} \text { such that } \forall a \in \mathcal{A}, d^{\top} M a \leq v .
$$

(Informally this states that if there exists a good choice of $d$ for each $a$ then there exists a good choice $d$ for all $a$.)

Now if a half-space, $\mathcal{H}=\left\{r \in \mathbb{R}^{n}: \hat{n} \cdot r \leq v\right\}$, that contains $\mathcal{Z}$ is approachable, then it must be that when the adversary choses $a \in \mathcal{A}$ there exists a choice $d \in \mathcal{D}$ such that

$$
\hat{n} \cdot R(d, a) \leq v .
$$

If this were not true then $\mathcal{H}$ certainly would not be approachable.

The statement (22) can be recast in terms of the Minimax Theorem. Specifically, we can define the matrix $M$ with components $M_{i j}:=\hat{n} \cdot R_{i j}$. Thus by definition $\hat{n} \cdot R(d, a)=d^{\top} M a$. Equation (22) now states that $\forall a \in \mathcal{A} \exists d \in \mathcal{D}$ such that

$$
d^{\top} M a \leq v
$$

Thus by the Minimax Theorem, (21), there exists $d \in \mathcal{D}$ such that

$$
\hat{n} \cdot R(d, a)=d^{\top} M a \leq v, \quad \forall a \in \mathcal{A} .
$$

So, we now know that for all hyperplanes $\mathcal{H}$ containing $\mathcal{Z}$, there exists a $d \in \mathcal{D}$ such that

$$
\hat{n} \cdot R(d, a) \in \mathcal{H}, \quad \forall a \in \mathcal{A} .
$$

This establishes the existence $d(t)$ as described in (6).

Next we show that the sequence $d(t), t \in \mathbb{Z}_{+}$is such that $\bar{Q}(t)$ approaches $\mathcal{Z}$.

$$
\begin{aligned}
& \|\bar{Q}(t+1)-P(t+1)\|^{2} \\
\leq & \|\bar{Q}(t+1)-P(t)\|^{2} \\
= & \|\bar{Q}(t+1)-\bar{Q}(t)\|^{2}+\|\bar{Q}(t)-P(t)\|^{2}+2(\bar{Q}(t+1)-\bar{Q}(t)) \cdot(\bar{Q}(t)-P(t)) \\
\leq & 2 \frac{R_{\max }^{2}}{(t+1)^{2}}+\|\bar{Q}(t)-P(t)\|^{2}+2(\bar{Q}(t+1)-\bar{Q}(t)) \cdot(\bar{Q}(t)-P(t)) .
\end{aligned}
$$


The first inequality holds since the projection $P(t+1)$ is by definition closer to $\bar{Q}(t+1)$. The equality is the parallelogram rule. The final inequality follows since, by the definition of $R_{\max }$ and since

$$
\bar{Q}(t+1)=\frac{1}{t+1} R(d(t+1), a(t+1))+\frac{t}{t+1} \bar{Q}(t) .
$$

Further, applying the above equality, to the final term in (24) gives

$$
\begin{aligned}
& (\bar{Q}(t+1)-\bar{Q}(t)) \cdot(\bar{Q}(t)-P(t)) \\
& =\frac{1}{t+1}\left(R(d(t+1), a(t+1))-\bar{Q}_{t}\right) \cdot(\bar{Q}(t)-P(\bar{Q}(t))) \\
& =\frac{1}{t+1}[(R(d(t+1), a(t+1))-P(t)) \cdot(\bar{Q}(t)-P(t))-(\bar{Q}(t)-P(t)) \cdot(\bar{Q}(t)-P(t))]
\end{aligned}
$$

Substituting (25) into (24) and taking expectations, we have

$$
\begin{aligned}
& \mathbb{E}\left[\|\bar{Q}(t+1)-P(t+1)\|^{2} \mid \mathcal{F}_{t}\right] \\
& \leq 2 \frac{R_{\max }^{2}}{(t+1)^{2}}+\left(1-\frac{2}{t+1}\right)\|\bar{Q}(t)-P(t)\|^{2}+\frac{2}{t+1}(R(\bar{d}(t+1), \bar{a}(t+1))-P(t)) \cdot(\bar{Q}(t)-P(t)) \\
& \leq 2 \frac{R_{\max }^{2}}{(t+1)^{2}}+\left(1-\frac{2}{t+1}\right)\|\bar{Q}(t)-P(\bar{Q}(t))\|^{2}
\end{aligned}
$$

where in the 1 st inequality above we note that

$$
\mathbb{E}\left[R(d(t+1), a(t+1)) \mid \mathcal{F}_{t}\right]=R(\bar{d}(t+1), \bar{a}(t+1))
$$

and in the 2nd inequality above we use the fact that

$$
(R(\bar{d}(t+1), \bar{a}(t+1))-P(t)) \cdot(\bar{Q}(t)-P(t)) \leq 0
$$

which holds by our assumption on $d(t),(6)$.

Multiplying both sides of $(26)$ by $(t+1)^{2}$ and rearranging gives

$$
(t+1)^{2} \mathbb{E}[\|\bar{Q}(t+1)-P(t+1)\|]^{2}-t^{2} \mathbb{E}\left[\|\bar{Q}(t)-P(t)\|^{2}\right] \leq 2 R_{\max }^{2}-\mathbb{E}\left[\|\bar{Q}(t)-P(t)\|^{2}\right] \leq 2 R_{\max }^{2} .
$$

Summing these interpolating terms gives

$$
t^{2} \mathbb{E}\left[\|\bar{Q}(t)-P(t)\|^{2}\right] \leq 2 R_{\max }^{2} t .
$$

Thus, as required,

$$
d(\bar{Q}(t), \mathcal{Z})=\sqrt{\mathbb{E}\left[\|\bar{Q}(t)-P(t)\|^{2}\right]} \leq R_{\max } \sqrt{\frac{2}{t}} \underset{t \rightarrow \infty}{\longrightarrow} 0
$$

\section{A.2 Hannan Gaddum Theorem}

Theorem 6 (Hannan-Gaddum Theorem). There exists a playing strategy $\left\{d_{t}\right\}_{t=1}^{\infty}$ such that for any $\left\{a_{t}\right\}_{t=1}^{\infty}$

$$
\limsup _{T \rightarrow \infty} \mathbb{E}\left[\frac{\mathcal{R} g(T)}{T}\right] \leq 0 .
$$

In other words, our performance in the game is asymptotically as good as the best fixed action.

The proof follows as a consequence of Blackwell's Approachability Theorem.

Proof. We define the vector payoff $R(d, a)=(r(i, a)-r(d, a): i=1, \ldots, p)$ and convex region $\mathcal{Z}=\{R$ : $\left.R_{i} \leq 0, \forall i=1, \ldots, p\right\}$. For all $a$ there exists $d$ such that component-wise $r(d, a) \leq 0$, in particular we choose $d$ to be the probability distribution with $d_{i^{*}}=1$ where $i^{*}=\operatorname{argmax}_{i=1, \ldots, p} r(i, a)$. This verifies Condition 2 of Blackwell's Approachability Theorem. Thus there exists a strategy $\{d(t)\}_{t=1}^{\infty}$ such that for all $\{a(t)\}_{t=1}^{\infty}$

$$
D(\mathcal{R g}(T) / T, \mathcal{Z}) \underset{T \rightarrow \infty}{\longrightarrow} 0
$$


As a consequence, we can analyse the expected regret:

$$
\begin{aligned}
\mathbb{E}\left[\frac{\mathcal{R} g(T)}{T}\right] & =\mathbb{E}\left[\max _{i=1, \ldots, p} \sum_{t=1}^{T} r(i, a(t))-\frac{1}{T} \sum_{t=1}^{T} r(d(t), a(t))\right] \\
& \leq \mathbb{E}\left[\sum_{i=1}^{m} \frac{1}{T} \sum_{t=1}^{T}|r(i, a(t))-r(d(t), a(t))|_{+}\right] \\
& \leq \mathbb{E}\left[\sum_{i=1}^{m} \frac{1}{T} \sum_{t=1}^{T}(r(i, a(t))-r(d(t), a(t)))_{+}^{2}\right]^{\frac{1}{2}} \\
& =D(\mathcal{R} g(T) / T, \mathcal{Z}) \underset{T \rightarrow \infty}{\longrightarrow} 0 .
\end{aligned}
$$

For first inequality above, we note that maximizing $i$ in (29) in included in the sum in (30). The second inequality follows from Jensen's inequality.

\section{A.3 Proof of MaxWeight Stability}

Theorem 7. Given $a(t), t \in \mathbb{Z}_{+}$are independent identically distributed with mean $\bar{a}$ then

i) If $\bar{a} \notin<\mathcal{S}>$ then, regardless of the policy used, $\left(Q_{j}(t): j=1, \ldots, q\right)$ is transient.

ii) If $\bar{a} \in<\mathcal{S}>^{\circ}$ then, under the MaxWeight policy, $\left(Q_{j}(t): j=1, \ldots, q\right)$ is positive recurrent.

Proof. For Part i), we note that given $\bar{a} \notin<\mathcal{S}>$ then there is a hyperplane separating $\bar{a}$ from $<\mathcal{S}>$. We let $\hat{n} \in \mathbb{Z}_{+}$be the normal vector of this hyperplane. Since this hyperplane is separating there exists an $\hat{\epsilon}$ such that

$$
\hat{n} \cdot(d-\bar{a}) \geq \hat{\epsilon}
$$

for all $d \in \mathcal{S}$. Thus we see that irrespective of the policy used it must hold that $\mathbb{E}[\hat{n} \cdot(Q(t)-Q(0))] \geq \hat{\epsilon} t$, which clearly diverges as $t \rightarrow \infty$. This proves Part i).

We now focus on Part ii). Here the crux of this argument is that for all queue sizes $q, q \cdot a$ is by some margin strictly less than the MaxWeight choice $\max _{\sigma \in<\mathcal{S}>q} \cdot \sigma$. Given $\epsilon$ above, we aim to use $\hat{\epsilon}$, the biggest such that $\bar{a}(t)+\hat{\epsilon} \mathbf{1} \in\langle\mathcal{S}>$ for all time. This can be defined as the biggest $\hat{\epsilon}$ such that $\hat{\epsilon} \leq \epsilon(t):=\max _{\sigma \in<\mathcal{S}>} \min _{j \in \mathcal{J}}\left(\sigma_{j}-\bar{a}_{j}(t)\right)$ for all $t \in \mathbb{Z}_{+}$i.e. so the distance of the smallest component to the boundary is maximized. Given this optimization description we observe the following

$$
\hat{\epsilon} \leq \max _{\sigma \in \mathcal{S}} \min _{j \in \mathcal{J}}\left(\sigma_{j}-\bar{a}_{j}(t)\right)=\max _{\sigma \in<\mathcal{S}>q: \sum_{j \in \mathcal{J}} q_{j}=1} q \cdot(\sigma-\bar{a}(t)) \leq \min _{q: \sum_{j \in \mathcal{J}} q_{j}=1} \max _{\sigma \in<\mathcal{S}>} q \cdot(\sigma-\bar{a}(t)) .{ }^{13}
$$

From this observation, we see one way that we can use $\hat{\epsilon}$ to bound the gap between $q \cdot \bar{a}(t)$ and $\max _{q \in<\mathcal{S}>q}$. $\sigma$.

$$
\begin{aligned}
& \mathbb{E}\|Q(t+1)\|^{2}-\mathbb{E}\|Q(t)\|^{2} \\
& =2 \mathbb{E}[Q(t) \cdot(a(t+1)-\sigma(t+1))]+\mathbb{E}\|a(t+1)-\sigma(t+1)\|^{2} \\
& =2 \mathbb{E}\left[Q^{\Sigma}(t) \frac{Q(t)}{Q^{\Sigma}(t)} \cdot(\bar{a}(t+1)-\sigma(t+1))\right]+2 \mathbb{E}[Q(t) \cdot(a(t+1)-\bar{a}(t+1))]+c \\
& \leq-2 \mathbb{E}\left[Q^{\Sigma}(t)\right] \min _{q: \sum_{j} q_{j}=1} \max _{\sigma \in<\mathcal{S}>} q \cdot(\sigma-\bar{a}(t+1))+c \\
& \leq-2 \mathbb{E}\left[Q^{\Sigma}(t)\right] \max _{\sigma \in<\mathcal{S}>\sum_{j} q_{j}=1} q \cdot(\sigma-\bar{a}(t+1))+c \\
& \leq-2 \hat{\epsilon} \mathbb{E}\left[Q^{\Sigma}(t)\right]+c .
\end{aligned}
$$

Summing the above expression from $t=0, \ldots, T-1$, we gain the expression

$$
-\mathbb{E}\|Q(0)\|^{2} \leq \mathbb{E}\|Q(T)\|^{2}-\mathbb{E}\|Q(0)\|^{2} \leq-2 \hat{\epsilon} \mathbb{E}\left[\sum_{t=0}^{T-1} Q^{\Sigma}(t)\right]+c T .
$$

\footnotetext{
${ }^{13}$ We inequality in the expression holds with equality by the Minimax Theorem; however, we only require this easier-to-prove inequality.
} 
Rearranging, dividing by $T$, and letting $T \rightarrow \infty$, we obtain the expression:

$$
\limsup _{T \rightarrow \infty} \mathbb{E}\left[\frac{1}{T} \sum_{t=0}^{T-1} Q^{\Sigma}(t)\right] \leq \frac{c}{2 \hat{\epsilon}} .
$$

which can only hold if the set of states $\left\{Q^{\Sigma}(t) \leq \frac{c}{2 \hat{\epsilon}}\right\}$ is positive recurrent.

\section{A.4 Regret and Service in a Queue}

Lemma 2. If $t_{0}$ is the last time before $t$ where the queue for policy $\pi$ is empty, i.e. $W_{t_{0}}^{\pi}=0$, then

$$
W_{t+1}^{\pi}-W_{t+1}^{\star} \leq \mathcal{R} g(t)-\mathcal{R} g\left(t_{0}-1\right) .
$$

Proof. Note that $Q_{t}^{\pi} \geq 0$ for $t \geq t_{0}$. Thus under the Lindley recursion it holds that

$$
W_{t+1}^{\pi}=W_{t}^{\pi}+\tau_{t}^{\pi}-a_{t}=\ldots=\sum_{s=t_{0}}^{t}\left(\tau_{s}^{\pi}-a_{s}\right) .
$$

Similarly repeated substitution for $W_{t}^{\pi}$ gives

$$
W_{t+1}^{\star}=0 \vee\left\{W_{t}^{\star}+\tau_{t}^{\star}-a_{t}\right\}=\ldots=\bigvee_{t^{\prime}=0}^{t}\left\{\sum_{s=t-t^{\prime}}^{t}\left(\tau_{s}^{\star}-a_{s}\right)\right\}
$$

The maximum above is less than the value attained at $t^{\prime}=t-t_{0}$ and so

$$
W_{t+1}^{\star} \geq \sum_{s=t_{0}}^{t}\left(\tau_{s}^{\star}-a_{s}\right)
$$

Combining the inequalities (34) and (35) then gives

$$
W_{t+1}^{\pi}-W_{t+1}^{\star} \leq \sum_{s=t_{0}}^{t}\left(\tau_{s}^{\pi}-\tau_{s}^{\star}\right)=\mathcal{R} g(t)-\mathcal{R} g\left(t_{0}-1\right)
$$

as required.

Proposition 3 (OCO bound). For any function $\nabla l_{t}(w)$ and $w_{t}$, as above, then for all $w \in \mathbb{R}^{p}$

$$
\sum_{t=1}^{T} l_{t}\left(w_{t}\right)-\sum_{t=1}^{T} l_{t}(w) \leq \frac{\left\|w_{1}-w\right\|}{\alpha_{1}}+\sum_{t=1}^{T} \frac{\left\|w_{t}-w\right\|^{2}}{2}\left(\frac{1}{\alpha_{t}}-\frac{1}{\alpha_{t+1}}\right)+\sum_{t=1}^{T} \frac{\alpha_{t}}{2}\left\|\nabla l_{t}\left(w_{t}\right)\right\|^{2} .
$$

Proof. For any $w \in \mathbb{R}^{p}$,

$$
\begin{aligned}
\left\|w_{t+1}-w\right\|^{2} & =\left\|w_{t}-\alpha_{t} \nabla l_{t}\left(w_{t}\right)-w\right\|^{2} \\
& \leq\left\|w_{t}-w\right\|^{2}+\alpha_{t}^{2}\left\|\nabla l_{t}\left(w_{t}\right)\right\|^{2}-2 \alpha_{t}\left(w_{t}-w\right) \cdot \nabla l_{t}\left(w_{t}\right) .
\end{aligned}
$$

Rearranging and summing gives

$$
\begin{aligned}
& \sum_{t=1}^{T}\left(w_{t}-w\right) \cdot \nabla l_{t}\left(w_{t}\right) \\
\leq & \sum_{t=1}^{T}\left[\frac{1}{2 \alpha_{t}}\left\|w_{t}-w\right\|^{2}-\frac{1}{2 \alpha_{t}}\left\|w_{t+1}-w\right\|^{2}\right]+\sum_{t=1}^{T} \frac{\alpha_{t}}{2}\left\|\nabla l_{t}\left(w_{t}\right)\right\|^{2} \\
= & -\frac{1}{2 \alpha_{T}}\left\|w_{T+1}-w\right\|^{2}+\sum_{t=1}^{T}\left\|w_{t}-w\right\|^{2}\left[\frac{1}{2 \alpha_{t}}-\frac{1}{2 \alpha_{t-1}}\right]+\sum_{t=1}^{T} \frac{\alpha_{t}}{2}\left\|\nabla l_{t}\left(w_{t}\right)\right\|^{2} \\
\leq & \sum_{t=1}^{T}\left\|w_{t}-w\right\|^{2}\left[\frac{1}{\alpha_{t}}-\frac{1}{\alpha_{t-1}}\right]+\sum_{t=1}^{T} \frac{\alpha_{t}}{2}\left\|\nabla l_{t}\left(w_{t}\right)\right\|^{2} .
\end{aligned}
$$


In the equality above we rearrange the order of summation in the square brackets (wlog. we take $\left.\left\|\theta_{1}-\theta\right\|^{2} / \alpha_{0}:=0\right)$. By convexity

$$
\sum_{t=1}^{T} l_{t}\left(w_{t}\right)-l_{t}(w) \leq \sum_{t=1}^{T}\left(w_{t}-w\right) \cdot \nabla l_{t}\left(w_{t}\right)
$$

The inequalities (36) and (37) gives the required bound.

Theorem 8 (Perceptron Mistake Bound). If there exists $w^{\star} \in \mathbb{R}^{p}$ such that $y^{(t)}\left\langle x^{(t)}, w^{\star}\right\rangle \geq 1$ then the perceptron algorithm is such that

$$
\sum_{t=1}^{\infty} \hat{l}_{t}\left(w_{t}\right) \leq D^{2}\left\|w^{\star}\right\|^{2}
$$

Proof. For $w^{\star}$ as stated, it holds that $\sum_{t=1}^{T} l_{t}\left(w^{\star}\right)=0$. Further, as discussed above, the loss $\hat{l}_{t}$ is less than the hinge loss $l_{t}$. With this and Proposition 2, it holds that

$$
\begin{aligned}
\sum_{t=1}^{T} \hat{l}_{t}\left(w_{t}\right) & \leq \sum_{t=1}^{T} l_{t}\left(w_{t}\right)-\sum_{t=1}^{T} l_{t}\left(w^{\star}\right) \\
& =\frac{\left\|w^{\star}\right\|^{2}}{2 \alpha}+\sum_{t=1}^{T} \frac{\left\|w_{t}-w^{\star}\right\|^{2}}{2}\left(\frac{1}{\alpha}-\frac{1}{\alpha}\right)+\sum_{t=1}^{T} \frac{\alpha}{2}\left\|\nabla l_{t}\left(w_{t}\right)\right\|^{2} \\
& =\frac{\left\|w^{\star}\right\|^{2}}{2 \alpha}+\frac{\alpha}{2} \sum_{t=1}^{T}\left\|y_{t} x_{t}\right\|^{2} \hat{l}_{t}\left(w_{t}\right) \\
& \leq \frac{\left\|w^{\star}\right\|^{2}}{2 \alpha}+\frac{\alpha}{2} D^{2} \sum_{t=1}^{T} \hat{l}_{t}\left(w_{t}\right)
\end{aligned}
$$

Notice that the number of mistakes is determined by the sign of $\langle w, x\rangle$ not the magnitude of $w$. This the $\sum_{t=1}^{T} \hat{l}_{t}(w)$ does not depend on $\alpha$. Optimizing over $\alpha$ in the bound (38) gives choice $\alpha=$ $\left\|w^{\star}\right\| /\left(D \sqrt{\sum_{t=1}^{T} \hat{l}_{t}}\right)$, which applying to the above inequality and rearranging yields:

$$
\sum_{t=1}^{T} \hat{l}_{t}\left(w_{t}\right) \leq\left\|w^{\star}\right\|^{2} D^{2}
$$

We can now let $T \rightarrow \infty$ for the required bound.

\section{Acknowledgement.}

The authors are grateful to Yash Kanoria making a number of helpful pointers and corrections to the results in Section 2. We are also thankful to two anonymous referees for their careful reading and their suggestions that have helped the structure and readability of the text.

\section{References}

S. Aalto, U. Ayesta, and R. Righter. On the gittins index in the m/g/1 queue. Queueing Systems, 63 (1):437-458, 2009.

J. Abernethy, P. L. Bartlett, and E. Hazan. Blackwell approachability and no-regret learning are equivalent. In Proceedings of the 24th Annual Conference on Learning Theory, pages 27-46. JMLR Workshop and Conference Proceedings, 2011.

S. Agrawal and R. Jia. Learning in structured mdps with convex cost functions: Improved regret bounds for inventory management. In Proceedings of the 2019 ACM Conference on Economics and Computation, pages 743-744, 2019.

J. Anselmi and F. Dufour. Power-of-d-choices with memory: Fluid limit and optimality. Mathematics of Operations Research, 45(3):862-888, 2020. 
C. Archer, S. Banerjee, M. Cortez, C. Rucker, S. R. Sinclair, M. Solberg, Q. Xie, and C. Yu. Orsuite: Benchmarking suite for sequential operations models. Reinforcement Learning in Networks and Queues, 2021.

G. Ash. Dynamic network evolution, with examples from at t's evolving dynamic network. IEEE Communications Magazine, 33(7):26-39, 1995. doi: 10.1109/35.391435.

B. Ata and X. Peng. An optimal callback policy for general arrival processes: a pathwise analysis. Operations Research, 68(2):327-347, 2020.

S. Banerjee, Y. Kanoria, and P. Qian. Dynamic assignment control of a closed queueing network under complete resource pooling. arXiv e-prints, pages arXiv-1803, 2018.

D. P. Bertsekas. Reinforcement learning and optimal control. Athena Scientific Belmont, MA, 2019.

O. Besbes, Y. Gur, and A. Zeevi. Non-stationary stochastic optimization. Operations research, 63(5): 1227-1244, 2015.

D. Blackwell. Controlled random walks. In Proceedings of the international congress of mathematicians, volume 3, pages 336-338, 1954.

D. Blackwell. An analog of the minimax theorem for vector payoffs. Pacific Journal of Mathematics, 6 (1):1-8, 1956.

A. Borodin, J. Kleinberg, P. Raghavan, M. Sudan, and D. P. Williamson. Adversarial queueing theory. In Proceedings of the twenty-eighth annual ACM symposium on Theory of computing, pages 376-385, 1996.

M. Bramson, B. D'Auria, and N. Walton. Stability and instability of the maxweight policy. arXiv preprint arXiv:1909.10825, 2019.

A. Cabrejas-Egea, R. Zhang, and N. Walton. Reinforcement learning for traffic signal control: Comparison with commercial systems. arXiv preprint arXiv:2104.10455, 2021.

N. Cesa-Bianchi and G. Lugosi. Prediction, learning, and games. Cambridge university press, 2006.

R. H. Crites and A. G. Barto. Improving elevator performance using reinforcement learning. Advances in neural information processing systems, pages 1017-1023, 1996.

J. Dai and M. Gluzman. Queueing network controls via deep reinforcement learning. arXiv preprint arXiv:2008.01644, 2020.

J. Dai and J. M. Harrison. Processing Networks: Fluid Models and Stability. Cambridge University Press, 2020.

J. G. Dai and W. Lin. Maximum pressure policies in stochastic processing networks. Operations Research, 53(2):197-218, 2005.

J. G. Dai and P. Shi. Inpatient overflow: An approximate dynamic programming approach. Manufacturing E Service Operations Management, 21(4):894-911, 2019.

A. Daniely, A. Gonen, and S. Shalev-Shwartz. Strongly adaptive online learning. In International Conference on Machine Learning, pages 1405-1411. PMLR, 2015.

A. Dieker, S. Ghosh, and M. S. Squillante. Optimal resource capacity management for stochastic networks. Operations Research, 65(1):221-241, 2017.

T. G. Dietterich. Editorial exploratory research in machine learning. Machine Learning, 5(1):5-9, 1990.

J. Feng, M. Gluzman, and J. Dai. Scalable deep reinforcement learning for ride-hailing. IEEE Control Systems Letters, 2020.

J. Gaitonde and É. Tardos. Stability and learning in strategic queuing systems. In Proceedings of the 21st ACM Conference on Economics and Computation, pages 319-347, 2020a.

J. Gaitonde and E. Tardos. Virtues of patience in strategic queuing systems. arXiv preprint arXiv:2011.10205, 2020b.

D. Gamarnik. Stability of adversarial queues via fluid models. In Proceedings 39th Annual Symposium on Foundations of Computer Science (Cat. No. 98CB36280), pages 60-70. IEEE, 1998.

D. Gamarnik, J. N. Tsitsiklis, and M. Zubeldia. Delay, memory, and messaging tradeoffs in distributed service systems. Stochastic Systems, 8(1):45-74, 2018.

D. Gamarnik, J. N. Tsitsiklis, M. Zubeldia, et al. A lower bound on the queueing delay in resource constrained load balancing. Annals of Applied Probability, 30(2):870-901, 2020.

L. Georgiadis, M. J. Neely, and L. Tassiulas. Resource allocation and cross-layer control in wireless networks. Foundations and Trends (R) in Networking, 1(1):1-144, 2006. 
R. J. Gibbens, F. P. Kelly, and P. Key. Dynamic alternative routing-modelling and behaviour. In Proceedings of the 12th International Teletraffic Congress, pages 1019-1025, 1988.

J. Gittins, K. Glazebrook, and R. Weber. Multi-armed bandit allocation indices. John Wiley \& Sons, 2011.

V. Gupta and A. Radovanović. Interior-point-based online stochastic bin packing. Operations Research, 68(5):1474-1492, 2020. doi: 10.1287/opre.2019.1914. URL https://doi.org/10.1287/opre.2019.1914.

E. Hazan. Introduction to online convex optimization. arXiv preprint arXiv:1909.05207, 2019.

T. Hellemans and B. Van Houdt. Performance analysis of load balancing policies with memory. In Proceedings of the 13th EAI International Conference on Performance Evaluation Methodologies and Tools, pages 27-34, 2020.

W.-K. Hsu, J. Xu, X. Lin, and M. R. Bell. Integrated online learning and adaptive control in queueing systems with uncertain payoffs. Operations Research, 2021.

Y. Hu, C. W. Chan, and J. Dong. Optimal scheduling of proactive service with customer deterioration and improvement. Manage. Sci.(forthcoming), 2020.

L. Huang, S. Zhang, M. Chen, and X. Liu. When backpressure meets predictive scheduling. IEEE/ACM Transactions on Networking, 24(4):2237-2250, 2015.

P. Jacko. Restless bandits approach to the job scheduling problem and its extensions. Modern trends in controlled stochastic processes: theory and applications, pages 248-267, 2010.

R. Johari, V. Kamble, and Y. Kanoria. Matching while learning. Operations Research, 2021.

Y. Kanoria and P. Qian. Blind dynamic resource allocation in closed networks via mirror backpressure. arXiv preprint arXiv:1903.02764, 2019.

S. Krishnasamy, R. Sen, R. Johari, and S. Shakkottai. Regret of queueing bandits. In D. Lee, M. Sugiyama, U. Luxburg, I. Guyon, and R. Garnett, editors, Advances in Neural Information Processing Systems, volume 29. Curran Associates, Inc., 2016. URL https://proceedings .neurips.cc/paper/2016/file/430c3626b879b4005d41b8a46172e0c0-Paper .pdf.

S. Krishnasamy, P. Akhil, A. Arapostathis, R. Sundaresan, and S. Shakkottai. Augmenting max-weight with explicit learning for wireless scheduling with switching costs. IEEE/ACM Transactions on Networking, 26(6):2501-2514, 2018.

S. Krishnasamy, R. Sen, R. Johari, and S. Shakkottai. Learning unknown service rates in queues: A multiarmed bandit approach. Operations Research, 69(1):315-330, 2021.

T. Lattimore and C. Szepesvári. Bandit algorithms. Cambridge University Press, 2020.

T. Le, P. Kovács, N. Walton, H. L. Vu, L. L. Andrew, and S. S. Hoogendoorn. Decentralized signal control for urban road networks. Transportation Research Part C: Emerging Technologies, 58: 431-450, 2015.

Q. Liang and E. Modiano. Minimizing queue length regret under adversarial network models. Proceedings of the ACM on Measurement and Analysis of Computing Systems, 2(1):1-32, 2018a.

Q. Liang and E. Modiano. Network utility maximization in adversarial environments. In IEEE INFOCOM 2018-IEEE Conference on Computer Communications, pages 594-602. IEEE, 2018b.

S. Lim, K. Jung, and M. Andrews. Stability of the max-weight protocol in adversarial wireless networks. IEEE/ACM Transactions on Networking, 22(6):1859-1872, 2013.

B. Liu, Q. Xie, and E. Modiano. Reinforcement learning for optimal control of queueing systems. In 2019 57th Annual Allerton Conference on Communication, Control, and Computing (Allerton), pages 663-670. IEEE, 2019.

X. Liu, B. Li, P. Shi, and L. Ying. Pond: Pessimistic-optimistic online dispatch. arXiv preprint arXiv:2010.09995, 2020.

C. Lopez, J. R. Marti, and S. Sarkaria. Distributed reinforcement learning in emergency response simulation. IEEE Access, 6:67261-67276, 2018.

X. Lu, B. Van Roy, V. Dwaracherla, M. Ibrahimi, I. Osband, and Z. Wen. Reinforcement learning, bit by bit. arXiv preprint arXiv:2103.04047, 2021.

S. T. Maguluri and R. Srikant. Heavy traffic queue length behavior in a switch under the maxweight algorithm. Stochastic Systems, 6(1):211-250, 2016. 
S. Mannor, R. Y. Rubinstein, and Y. Gat. The cross entropy method for fast policy search. In Proceedings of the 20th International Conference on Machine Learning (ICML-03), pages 512-519, 2003.

L. Massoulié and $\mathrm{K}$. Xu. On the capacity of information processing systems. Operations Research, 66 (2):568-586, 2018.

N. McKeown, A. Mekkittikul, V. Anantharam, and J. Walrand. Achieving 100\% throughput in an input-queued switch. IEEE Transactions on Communications, 47(8):1260-1267, 1999.

M. Mitzenmacher, B. Prabhakar, and D. Shah. Load balancing with memory. In The 43rd Annual IEEE Symposium on Foundations of Computer Science, 2002. Proceedings., pages 799-808. IEEE, 2002.

O. Morgenstern and J. Von Neumann. Theory of games and economic behavior. Princeton university press, 1953.

M. J. Neely. Stochastic network optimization with application to communication and queueing systems. Synthesis Lectures on Communication Networks, 3(1):1-211, 2010a.

M. J. Neely. Universal scheduling for networks with arbitrary traffic, channels, and mobility. In 49th IEEE Conference on Decision and Control (CDC), pages 1822-1829. IEEE, 2010b.

M. J. Neely, E. Modiano, and C.-P. Li. Fairness and optimal stochastic control for heterogeneous networks. IEEE/ACM Transactions On Networking, 16(2):396-409, 2008.

M. J. Neely, S. T. Rager, and T. F. La Porta. Max weight learning algorithms for scheduling in unknown environments. IEEE Transactions on Automatic Control, 57(5):1179-1191, 2012.

A. B. Novikoff. On convergence proofs for perceptrons. Technical report, STANFORD RESEARCH INST MENLO PARK CA, 1963.

Y. Pan, Z. Xu, J. Guang, J. Sun, C. Wang, X. Zhang, X. Chen, J. Dai, Y. Ding, P. Shi, et al. A highfidelity, machine-learning enhanced queueing network simulation model for hospital ultrasound operations. arXiv preprint arXiv:2104.07451, 2021.

I.-B. Park, J. Huh, J. Kim, and J. Park. A reinforcement learning approach to robust scheduling of semiconductor manufacturing facilities. IEEE Transactions on Automation Science and Engineering, 17(3):1420-1431, 2019.

K. Prabuchandran, T. Bodas, and T. Tulabandhula. Reinforcement learning algorithms for regret minimization in structured markov decision processes. arXiv preprint arXiv:1608.04929, 2016.

Z. Qin, J. Tang, and J. Ye. Deep reinforcement learning with applications in transportation. In Proceedings of the 25th ACM SIGKDD International Conference on Knowledge Discovery \& Data Mining, pages 3201-3202, 2019.

G. Qu, A. Wierman, and N. Li. Scalable reinforcement learning of localized policies for multi-agent networked systems. In Learning for Dynamics and Control, pages 256-266. PMLR, 2020.

S. Qu, J. Wang, and J. Jasperneite. Dynamic scheduling in large-scale stochastic processing networks for demand-driven manufacturing using distributed reinforcement learning. In 2018 IEEE 23rd International Conference on Emerging Technologies and Factory Automation (ETFA), volume 1, pages 433-440. IEEE, 2018.

A. N. Rybko and A. L. Stolyar. Ergodicity of stochastic processes describing the operation of open queueing networks. Problemy Peredachi Informatsii, 28(3):3-26, 1992.

Z. Scully, M. Harchol-Balter, and A. Scheller-Wolf. Simple near-optimal scheduling for the $\mathrm{m} / \mathrm{g} / 1$. Proceedings of the ACM on Measurement and Analysis of Computing Systems, 4(1):1-29, 2020.

D. Shah, D. Wischik, et al. Switched networks with maximum weight policies: Fluid approximation and multiplicative state space collapse. The Annals of Applied Probability, 22(1):70-127, 2012.

D. Shah, Q. Xie, and Z. Xu. Stable reinforcement learning with unbounded state space. In Learning for Dynamics and Control, pages 581-581. PMLR, 2020a.

V. Shah, L. Gulikers, L. Massoulié, and M. Vojnović. Adaptive matching for expert systems with uncertain task types. Operations Research, 68(5):1403-1424, $2020 \mathrm{~b}$.

S. Shalev-Shwartz et al. Online learning and online convex optimization. Foundations and trends in Machine Learning, 4(2):107-194, 2011.

A. Sharifnassab, J. N. Tsitsiklis, and S. J. Golestani. Fluctuation bounds for the max-weight policy with applications to state space collapse. Stochastic Systems, 10(3):223-250, 2020.

S. Singh and D. Bertsekas. Reinforcement learning for dynamic channel allocation in cellular telephone systems. Advances in neural information processing systems, pages 974-980, 1997. 
S. Smith, G. Barlow, X.-F. Xie, and Z. Rubinstein. Smart urban signal networks: Initial application of the surtrac adaptive traffic signal control system. In Proceedings of the International Conference on Automated Planning and Scheduling, volume 23, 2013.

J. Spencer, M. Sudan, and K. Xu. Queueing with future information. ACM SIGMETRICS Performance Evaluation Review, 41(3):40-42, 2014.

T. Stahlbuhk, B. Shrader, and E. Modiano. Learning algorithms for scheduling in wireless networks with unknown channel statistics. Ad Hoc Networks, 85:131-144, 2019.

S. Stidham. Optimal control of admission to a queueing system. IEEE Transactions on Automatic Control, 30(8):705-713, 1985.

A. L. Stolyar et al. Maxweight scheduling in a generalized switch: State space collapse and workload minimization in heavy traffic. Annals of Applied Probability, 14(1):1-53, 2004.

R. S. Sutton and A. G. Barto. Reinforcement learning: An introduction. MIT press, 2018.

C. Szepesvári. Algorithms for reinforcement learning. Synthesis lectures on artificial intelligence and machine learning, 4(1):1-103, 2010.

L. Tassiulas and A. Ephremides. Stability properties of constrained queueing systems and scheduling policies for maximum throughput in multihop radio networks. In 29th IEEE Conference on Decision and Control, pages 2130-2132. IEEE, 1990.

G. Tesauro, R. Das, W. E. Walsh, and J. O. Kephart. Utility-function-driven resource allocation in autonomic systems. In Second International Conference on Autonomic Computing (ICAC'05), pages 342-343. IEEE, 2005a.

G. Tesauro, N. K. Jong, R. Das, and M. N. Bennani. A hybrid reinforcement learning approach to autonomic resource allocation. In 2006 IEEE International Conference on Autonomic Computing, pages 65-73. IEEE, 2006.

G. Tesauro et al. Online resource allocation using decompositional reinforcement learning. In $A A A I$, volume 5, pages 886-891, 2005b.

C. Tessler, Y. Shpigelman, G. Dalal, A. Mandelbaum, D. H. Kazakov, B. Fuhrer, G. Chechik, and S. Mannor. Reinforcement learning for datacenter congestion control. arXiv preprint arXiv:2102.0933\%, 2021.

H. Topcuoglu, S. Hariri, and M.-Y. Wu. Performance-effective and low-complexity task scheduling for heterogeneous computing. IEEE transactions on parallel and distributed systems, 13(3):260-274, 2002.

T. van Barneveld, C. Jagtenberg, S. Bhulai, and R. van der Mei. Real-time ambulance relocation: Assessing real-time redeployment strategies for ambulance relocation. Socio-Economic Planning Sciences, 62:129-142, 2018.

B. Van Roy, D. P. Bertsekas, Y. Lee, and J. N. Tsitsiklis. A neuro-dynamic programming approach to retailer inventory management. In Proceedings of the 36th IEEE Conference on Decision and Control, volume 4, pages 4052-4057. IEEE, 1997.

V. Vapnik. The nature of statistical learning theory. Springer science \& business media, 2013.

P. Varaiya. Max pressure control of a network of signalized intersections. Transportation Research Part C: Emerging Technologies, 36:177-195, 2013.

N. S. Walton. Two queues with non-stochastic arrivals. Operations Research Letters, 42(1):53-57, 2014.

N. Xiao, E. Frazzoli, Y. Luo, Y. Li, Y. Wang, and D. Wang. Throughput optimality of extended backpressure traffic signal control algorithm. In 2015 23rd Mediterranean Conference on Control and Automation (MED), pages 1059-1064. IEEE, 2015.

K. Xu. Necessity of future information in admission control. Operations Research, 63(5):1213-1226, 2015.

K. Xu and C. W. Chan. Using future information to reduce waiting times in the emergency department via diversion. Manufacturing \& Service Operations Management, 18(3):314-331, 2016.

$\mathrm{K}$. Xu and Y. Zhong. Information and memory in dynamic resource allocation. Operations Research, 68 (6):1698-1715, 2020.

M. Zaki, A. Mohan, A. Gopalan, and S. Mannor. Better than the best: Gradient-based improper reinforcement learning for network scheduling. arXiv preprint arXiv:2105.00210, 2021a.

M. Zaki, A. Mohan, A. Gopalan, and S. Mannor. Improper learning with gradient-based policy optimization. arXiv preprint arXiv:2102.08201, 2021b. 
W. Zhang and T. G. Dietterich. A reinforcement learning approach to job-shop scheduling. In IJCAI, volume 95, pages 1114-1120. Citeseer, 1995. 